\title{
COHERENT STATES ON SPHERES
}

\author{
BRIAN C. HALL AND JEFFREY J. MITCHELL
}

\begin{abstract}
We describe a family of coherent states and an associated resolution of the identity for a quantum particle whose classical configuration space is the $d$-dimensional sphere $S^{d}$. The coherent states are labeled by points in the associated phase space $T^{*}\left(S^{d}\right)$. These coherent states are not of Perelomov type but rather are constructed as the eigenvectors of suitably defined annihilation operators.

We describe as well the Segal-Bargmann representation for the system, the associated unitary Segal-Bargmann transform, and a natural inversion formula. Although many of these results are in principle special cases of the results of B. Hall and M. Stenzel, we give here a substantially different description based on ideas of T. Thiemann and of K. Kowalski and J. Rembieliński.

All of these results can be generalized to a system whose configuration space is an arbitrary compact symmetric space. We focus on the sphere case in order to carry out the calculations in a self-contained and explicit way.
\end{abstract}

\section{Contents}

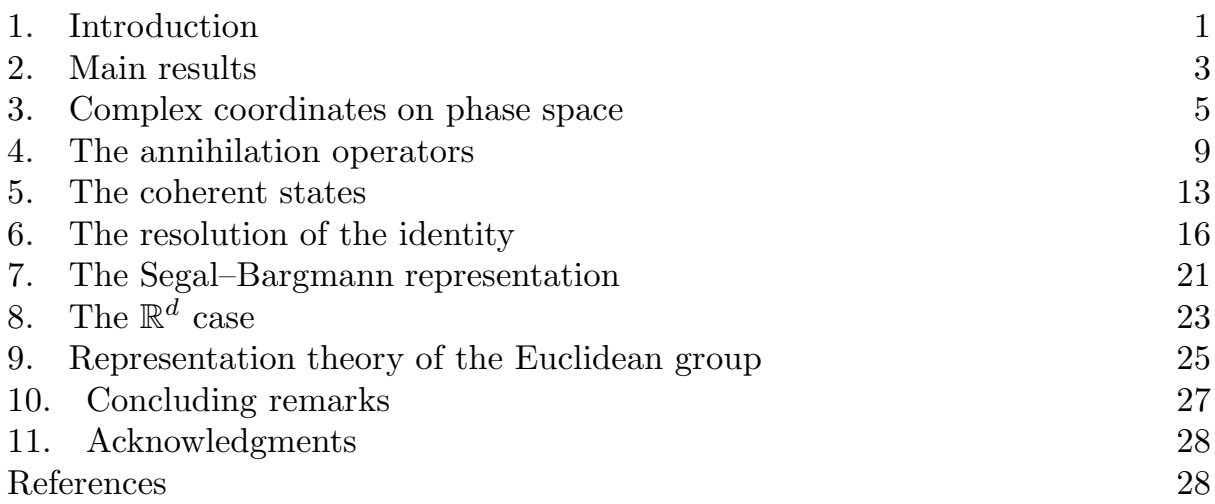

\section{INTRODUCTION}

In [1] B. Hall introduced a family of coherent states for a system whose classical configuration space is the group manifold of a compact Lie group $G$. These coherent states are labeled by points in the associated phase space, namely the cotangent bundle $T^{*}(G)$. The coherent states themselves were originally defined in terms of the heat kernel on $G$, although we will give a different perspective here. One may identify [2] $T^{*}(G)$ with the complexified group $G_{\mathbb{C}}$, where, for example, if $G=\mathrm{SU}(2)$ then $G_{\mathbb{C}}=\operatorname{SL}(2 ; \mathbb{C})$. The paper [1] establishes a resolution of the identity for these coherent states, and equivalently, a unitary Segal-Bargmann transform. The SegalBargmann representation of this system is a certain Hilbert space of holomorphic 
functions over the complex group $G_{\mathbb{C}}$. Additional results may be found in [2, 3, 田 and the survey paper $[5]$.

The coherent states for $G$ (in the form of the associated Segal-Bargmann transform) have been applied to quantum gravity in [6], with proposed generalizations due to Thiemann [7]. More recently the coherent states themselves have been used by Thiemann and co-authors [8] in an attempt to determine the classical limit of the quantum gravity theory proposed by Thiemann in [9]. In particular, the second entry in [8] establishes good phase space localization properties (in several different senses) for the coherent states associated to the configuration space $G=\mathrm{SU}(2)$.

In another direction, K. K. Wren [10], using a method proposed by N. P. Landsman 11, has shown that the coherent states for $G$ arise naturally in the canonical quantization of $(1+1)$-dimensional Yang-Mills theory on a spacetime cylinder. Here $G$ is the structure group of the theory and plays the role of the reduced configuration space, that is, the space of connections modulo based gauge transformation over the spatial circle. Wren considers first the ordinary canonical coherent states for the unreduced (infinite-dimensional) system. He then shows that after "projecting" them into the gauge-invariant subspace (using a suitable regularization procedure) these become precisely the generalized coherent states for $G$, as originally defined in [1]. Driver and Hall [12] elaborate on the results of Wren, using a different regularization scheme. They show in particular how the resolution of the identity for the generalized coherent states can be obtained by projection from the resolution of the identity for the canonical coherent states. See also 13] for an appearance of the generalized Segal-Bargmann transform in the setting of 2-dimensional Euclidean Yang-Mills theory.

Finally, the paper [14] shows that the generalized Segal-Bargmann transform for $G$ can be obtained by means of geometric quantization (see also [5, Sect. 3.2]). This means that the associated coherent states for $G$ are of "Rawnsley type" 15 and are thus in the spirit of Berezin's approach to quantization.

We emphasize that the coherent states for $G$ are not of Perelomov type [16]. Instead they are realized as the eigenvectors of certain non-self-adjoint "annihilation operators," as will be described in detail in the present paper. (See Section 10 for further comments.)

The coherent states and the resolution of the identity for $G$ "descend" is a straightforward way to the case of a system whose configuration space is a compact symmetric space $X$ [1, Sect. 11]. Compact symmetric spaces are manifolds of the form $G / K$, where $G$ is a compact Lie group and $K$ is a special sort of subgroup, namely, the fixed-point subgroup of an involution. Examples include the spheres $S^{d}=\mathrm{SO}(d+1) / \mathrm{SO}(d)$ and the complex projective spaces $\mathbb{C} P^{d}=\mathrm{SU}(d+1) / \mathrm{SU}(d)$. Compact Lie groups themselves can be thought of as symmetric spaces by identifying $G$ with $(G \times G) / \Delta(G)$ where $\Delta(G)$ is the "diagonal" copy of $G$ inside $G \times G$.

We emphasize that in the case $X=S^{2}$ the 2-sphere is playing the role of the configuration space and thus the coherent states discussed here are completely different from the spin coherent states in which the 2 -sphere plays the role of the phase space. Whereas the spin coherent states are labeled by points in $S^{2}$ itself, our coherent states are labeled by points in the cotangent bundle $T^{*}\left(S^{2}\right)$.

Although the case of compact symmetric spaces can be treated by descent from the group, it is preferable to give a direct treatment, and such a treatment was given by Stenzel 177. In particular Stenzel gives a much better description, in the symmetric space case, of the measure that one uses to construct the resolution 
of the identity. (See also [5, Sect. 3.4].) Although Stenzel formulates things in terms of a unitary Segal-Bargmann transform and does not explicitly mention the coherent states, only a notational change is needed to re-express his results as a resolution of the identity for the associated coherent states.

More recently, the coherent states for the 2-sphere $S^{2}$ were independently discovered, from a substantially different point of view, by Kowalski and Rembieliński [18. (The paper [18] builds on earlier work of Kowalski, Rembieliński, and Papaloucas [19] on the $S^{1}$ case.) These authors were unaware at the time of the work of Hall and Stenzel. The paper [20] then describes the resolution of the identity for the coherent states on the 2-sphere, showing in a different and more explicit way that the result of [17, Thm. 3] holds in this case. (See Section VII of [20] for comments on the relation of their work to that of Stenzel.)

The purpose of this paper is to describe the coherent states for a compact symmetric space using the points of view advocated by Thiemann and by Kowalski and Rembieliński. For the sake of concreteness we concentrate in this paper on the case $X=S^{d}$. In [1] and [17 the coherent states are defined in terms of the heat kernel on the configuration space, which takes the place of the Gaussian that enters into the description of the canonical coherent states. Here by contrast the coherent states are defined to be the eigenvectors of suitable annihilation operators, and only afterwards does one discover the role of the heat kernel, in the position wave function of the coherent states and in the reproducing kernel. The annihilation operators, meanwhile, are defined by (a special case of) the "complexifier" method proposed by Thiemann, which we will show is equivalent to (a generalization of) the polardecomposition method of Kowalski and Rembieliński. We emphasize, though, that the approach described in this paper gives ultimately the same results as the heat kernel approach of Hall and Stenzel.

\section{MAin RESUlts}

In this section we briefly summarize the main results of the paper. All results are explained in greater detail in the subsequent sections. Briefly, our strategy is this. First, we construct complex-valued functions $a_{1}, \cdots, a_{d+1}$ on the classical phase space that serve to define a complex structure on phase space. Second, we construct the quantum counterparts of these functions, operators $A_{1}, \cdots, A_{d+1}$ that we regard as the annihilation operators. Third, we construct simultaneous eigenvectors for the annihilation operators, which we regard as the coherent states. Fourth, we construct a resolution of the identity for these coherent states.

We consider a system whose classical configuration space is the $d$-dimensional sphere $S^{d}$ of radius $r$. We consider also the corresponding phase space, the cotangent bundle $T^{*}\left(S^{d}\right)$, which we describe as

$$
T^{*}\left(S^{d}\right)=\left\{(\mathbf{x}, \mathbf{p}) \in \mathbb{R}^{d+1} \times \mathbb{R}^{d+1} \mid x^{2}=r^{2}, \mathbf{x} \cdot \mathbf{p}=0\right\},
$$

where $\mathbf{p}$ is the linear momentum.

In Section 3 we consider the classical component of Thiemann's method. To apply this method we must choose a constant $\omega$ with units of frequency. The classical "complexifier" is then defined to be kinetic energy function divided by $\omega$, which can be expressed as

$$
\text { complexifier }=\frac{\text { kinetic energy }}{\omega}=\frac{j^{2}}{2 m \omega r^{2}},
$$


where $j^{2}$ is the total angular momentum. (Thiemann's method allows other complexifiers; see Section 3.) We construct complex-valued functions $a_{1}, \cdots, a_{d+1}$ by taking the position functions $x_{1}, \cdots, x_{d+1}$ and applying repeated Poisson brackets with the complexifier. Specifically,

$$
\begin{aligned}
a_{k} & =e^{i\{\cdot, \text { complexifier }\}} x_{k} \\
& =\sum_{n=0}^{\infty}\left(\frac{i}{2 m \omega r^{2}}\right)^{n} \frac{1}{n !} \underbrace{\left\{\cdots\left\{\left\{x_{k}, j^{2}\right\}, j^{2}\right\}, \cdots, j^{2}\right\}}_{n}
\end{aligned}
$$

If we let $\mathbf{a}=\left(a_{1}, \cdots, a_{d+1}\right)$ then the calculations in Section 3 will give the following explicit formula

$$
\mathbf{a}=\cosh \left(\frac{j}{m \omega r^{2}}\right) \mathbf{x}+i \frac{r^{2}}{j} \sinh \left(\frac{j}{m \omega r^{2}}\right) \mathbf{p} .
$$

These complex-valued functions satisfy $a_{1}^{2}+\cdots+a_{d+1}^{2}=r^{2}$ and $\left\{a_{k}, a_{l}\right\}=0$. In the case $d=2$ this agrees with Eq. (6.1) of [18].

In Section 1 we consider the quantum component of Thiemann's method. We consider the quantum counterpart of the classical complexifier, namely,

$$
\text { complexifier }=\frac{\text { kinetic energy }}{\omega}=\frac{J^{2}}{2 m \omega r^{2}},
$$

where $J^{2}$ is the total angular momentum operator. Then if $X_{1}, \cdots, X_{d+1}$ denote the position operators we define, by analogy with (1),

$$
\begin{aligned}
A_{k} & =e^{i[\cdot, \text { complexifier }] / i \hbar} X_{k} \\
& =\sum_{n=0}^{\infty}\left(\frac{1}{2 m \omega r^{2} \hbar}\right)^{n} \frac{1}{n !} \underbrace{\left[\cdots\left[\left[X_{k}, J^{2}\right], J^{2}\right], \cdots, J^{2}\right]}_{n} .
\end{aligned}
$$

This may also be written as

$$
A_{k}=e^{-J^{2} / 2 m \omega r^{2} \hbar} X_{k} e^{J^{2} / 2 m \omega r^{2} \hbar} .
$$

Equation (30) in Section 1 gives the quantum counterpart of (2); it is slightly more complicated than (2) because of quantum corrections. The annihilation operators satisfy $A_{1}^{2}+\cdots+A_{d+1}^{2}=r^{2}$ and $\left[A_{k}, A_{l}\right]=0$. Applying the same procedure in the $\mathbb{R}^{d}$ case produces the usual complex coordinates on phase space and the usual annihilation operators (Section 8).

One can easily deduce from (位 a "polar decomposition" for the annihilation operator, given in (33) in Section 1 . In the case $d=2$ this is essentially the same as what Kowalski and Rembieliński take as the definition of the annihilation operators. This shows that Thiemann's complexifier approach is equivalent to the polar decomposition approach of Kowalski and Rembieliński. Similarly, the polar form of the annihilation operators in the $d=1$ case is essentially the same as what Kowalski, Rembieliński, and Papaloucas take as the definition of the annihilation operator in 19 .

In Section 5 we consider the coherent states, defined to be the simultaneous eigenvectors of the annihilation operators. Using (4) we may immediately write down some eigenvectors for the $A_{k}$ 's, namely, the vectors of the form

$$
\left|\psi_{\mathbf{a}}\right\rangle=e^{-J^{2} / 2 m \omega r^{2} \hbar}\left|\delta_{\mathbf{a}}\right\rangle
$$


where $\left|\delta_{\mathbf{a}}\right\rangle$ is a simultaneous eigenvector for the position operators corresponding to a point $\mathbf{a}$ in $S^{d}$. A key result of Section 5 is that one can perform an analytic continuation with respect to the parameter a, thereby obtaining coherent states $\left|\psi_{\mathbf{a}}\right\rangle$ corresponding to any point $\mathbf{a}$ in the complexified sphere, $S_{\mathbb{C}}^{d}=\left\{\mathbf{a} \in \mathbb{C}^{d+1} \mid a^{2}=r^{2}\right\}$. These vectors $\left|\psi_{\mathbf{a}}\right\rangle$ are normalizable and satisfy

$$
A_{k}\left|\psi_{\mathbf{a}}\right\rangle=a_{k}\left|\psi_{\mathbf{a}}\right\rangle, \quad \mathbf{a} \in S_{\mathbb{C}}^{d}
$$

Equation (5) shows that the coherent states are expressible in terms of the heat kernel on the sphere, thus demonstrating that Thiemann's definition of the coherent states is equivalent to the definition in [1, 17] in terms of the heat kernel. The reproducing kernel for these coherent states is also expressed in terms of the heat kernel on the sphere.

In Section 6 we describe a resolution of the identity for these coherent states. In a suitable coordinate system this takes the form

$$
I=\int_{\mathbf{x} \in S^{d}} \int_{\mathbf{p} \cdot \mathbf{x}=0}\left|\psi_{\mathbf{a}}\right\rangle\left\langle\psi_{\mathbf{a}}\right| \nu(2 \tau, 2 p)\left(\frac{\sinh 2 p}{2 p}\right)^{d-1} 2^{d} d \mathbf{p} d \mathbf{x}
$$

where $\mathbf{a}$ is a function of $\mathbf{x}$ and $\mathbf{p}$ as in (2). Here $\nu$ is the heat kernel for $d$-dimensional hyperbolic space and $\tau$ is the dimensionless quantity given by $\tau=\hbar / m \omega r^{2}$. Explicit formulas for $\nu$ are found in Section 6 . The resolution of the identity for the coherent states is obtained by a continuous deformation of the resolution of the identity for the position eigenvectors.

In Section 7 we discuss the Segal-Bargmann representation for this system, namely, the space of holomorphic functions on the complexified sphere that are square-integrable with respect to the density in (6). We think of the Segal-Bargmann representation as giving a sort of phase space wave function for any state. There is an inversion formula stating the position wave function can be obtained from the phase space wave function by integrating out the momentum variables, specifically,

$$
\left\langle\delta_{\mathbf{x}} \mid \phi\right\rangle=\int_{\mathbf{p} \cdot \mathbf{x}=0}\left\langle\psi_{\mathbf{a}(\mathbf{x}, \mathbf{p})} \mid \phi\right\rangle \nu(\tau, p)\left(\frac{\sinh p}{p}\right)^{d-1} d \mathbf{p}
$$

for any state $|\phi\rangle$. Note that whereas the resolution of the identity involves $\nu(2 \tau, 2 p)$, the inversion formula involves $\nu(\tau, p)$.

In Section 8 we show that the complexifier method, when applied to the $\mathbb{R}^{d}$ case, yields the usual canonical coherent states and their resolution of the identity. In Section 9 we summarize some of the relevant representation theory for the Euclidean group. Finally, in Section 10 we compare our construction to other constructions of coherent states on spheres.

Although all of the results here generalize to arbitrary compact symmetric spaces $X$, we concentrate for the sake of explicitness on the case $X=S^{d}$. We will describe the general case in a forthcoming paper.

\section{Complex coordinates on phase space}

In this section we define Poisson-commuting complex-valued functions $a_{1}, \cdots, a_{d+1}$ on the classical phase space. In Section 1 we will introduce the quantum counterparts of these functions, commuting non-self-adjoint operators $A_{1}, \cdots, A_{d+1}$ which we regard as the annihilation operators for this system. In Section 5 we will consider the coherent states, that is, the simultaneous eigenvectors of the annihilation operators. 
Consider the $d$-dimensional sphere of radius $r$ in $\mathbb{R}^{d+1}$, namely,

$$
S^{d}=\left\{\mathbf{x} \in \mathbb{R}^{d+1} \mid x_{1}^{2}+\cdots+x_{d+1}^{2}=r^{2}\right\},
$$

regarded as the configuration space for a classical system $(d \geq 1)$. Then consider the associated phase space, the cotangent bundle $T^{*}\left(S^{d}\right)$, which we think of as

$$
T^{*}\left(S^{d}\right)=\left\{(\mathbf{x}, \mathbf{p}) \in \mathbb{R}^{d+1} \times \mathbb{R}^{d+1} \mid x^{2}=r^{2}, \mathbf{x} \cdot \mathbf{p}=0\right\} .
$$

Here $p$ is the linear momentum, which must be tangent to $S^{d}$, i.e. perpendicular to $\mathbf{x}$.

We also have the angular momentum functions $j_{k l}, 1 \leq k, l \leq d+1$, given by

$$
j_{k l}=p_{k} x_{l}-p_{l} x_{k} .
$$

We may think of $j$ as a function on $T^{*}\left(S^{d}\right)$ taking values in the space of $(d+1) \times$ $(d+1)$ skew-symmetric matrices, that is, in the Lie algebra so $(d+1)$. Thinking of $j$ as a matrix we may re-write (7) as

$$
\mathbf{j}(\mathbf{x}, \mathbf{p})=\mathbf{p} \otimes \mathbf{x}-\mathbf{x} \otimes \mathbf{p},
$$

where $\otimes$ denotes the outer product. (That is, $(\mathbf{a} \otimes \mathbf{b})_{k l}=a_{k} b_{l}$.)

For a particle constrained to the sphere it is possible and convenient to express everything in terms of $\mathbf{x}$ and $\mathbf{j}$ instead of $\mathbf{x}$ and $\mathbf{p}$. We may alternatively describe $T^{*}\left(S^{d}\right)$ as the set of pairs $(\mathbf{x}, \mathbf{j})$ in which $\mathbf{x}$ is a vector in $\mathbb{R}^{d+1}, \mathbf{j}$ is a $(d+1) \times(d+1)$ skew-symmetric matrix, and $\mathbf{x}$ and $\mathbf{j}$ satisfy

$$
x^{2}=r^{2}
$$

and

$$
r^{2} j_{k l}=j_{k m} x_{m} x_{l}-x_{k} j_{l m} x_{m}
$$

(sum convention). This last condition says that if we define $\mathbf{p}$ to be $r^{-2} \mathbf{j} \mathbf{x}$, then $\mathbf{j}=\mathbf{p} \otimes \mathbf{x}-\mathbf{x} \otimes \mathbf{p}$. Equation (9) reflects the constraint to the sphere and does not hold for a general particle in $\mathbb{R}^{d+1}$. On $T^{*}\left(S^{d}\right)$ we have the relations

$$
\begin{aligned}
& \mathbf{j} \mathbf{x}=r^{2} \mathbf{p} \\
& \mathbf{j p}=-p^{2} \mathbf{x} .
\end{aligned}
$$

Recall that $\mathbf{j}$ is a matrix; thus $\mathbf{j} \mathbf{x}$ is the vector obtained by applying the matrix $\mathbf{j}$ to the vector $\mathbf{x}$, and similarly for $\mathbf{j p}$.

In the case $d=2\left(S^{2}\right.$ sitting inside $\left.\mathbb{R}^{3}\right)$ a standard vector identity shows that for any vector $\mathbf{v} \in \mathbb{R}^{3}, \mathbf{j} \mathbf{v}=(\mathbf{x} \times \mathbf{p}) \times \mathbf{v}$, where $\times$ is the cross-product and $\mathbf{x} \times \mathbf{p}$ is the usual angular momentum vector $\mathbf{l}$. So in the $\mathbb{R}^{3}$ case $\mathbf{j} \mathbf{v}=\mathbf{l} \times \mathbf{v}$.

The symplectic structure on $T^{*}\left(S^{d}\right)$ may be characterized by the Poisson bracket relations

$$
\begin{aligned}
\left\{j_{k l}, j_{m n}\right\} & =\delta_{k n} j_{l m}+\delta_{l m} j_{k n}-\delta_{k m} j_{l n}-\delta_{l n} j_{k m} \\
\left\{x_{k}, j_{l m}\right\} & =\delta_{k l} x_{m}-\delta_{k m} x_{l} \\
\left\{x_{k}, x_{l}\right\} & =0
\end{aligned}
$$

These are the commutation relations for the Euclidean Lie algebra, which is the semidirect product $\mathrm{e}(d+1) \cong \operatorname{so}(d+1) \ltimes \mathbb{R}^{d+1}$.

Poisson bracket relations involving $\mathbf{p}$ should be derived from (11) using the relation $\mathbf{p}=r^{-2} \mathbf{j x}$. Since the constraint to the sphere alters the dynamics and 
hence the Poisson bracket relations, we will not get the same formulas as in $\mathbb{R}^{d+1}$. For example, we have

$$
\left\{x_{k}, p_{l}\right\}=\delta_{k l}-\frac{x_{k} x_{l}}{r^{2}} .
$$

The complex coordinates on phase space will be constructed from the position functions $x_{k}$ by means of repeated Poisson brackets with a multiple of the kinetic energy function. In the sphere case it is convenient to express the kinetic energy in terms of the total angular momentum $j^{2}$, given by

$$
j^{2}=\sum_{k<l}\left(j_{k l}\right)^{2} \text {. }
$$

The total angular momentum satisfies $j^{2}=r^{2} p^{2}$, and the kinetic energy is $p^{2} / 2 m=$ $j^{2} / 2 m r^{2}$.

We now choose a constant $\omega$ with units of a frequency. The significance of this constant is that $m \omega$ has the units of momentum divided by position. Thus $\omega$ (together with $m$ ) allows us to put position and momentum onto the same scale, which is necessary in order define complex-valued functions that involve both $x$ and $p$. Ultimately, $m \omega$ will control the ratio of the width in position space of the coherent states to the radius of the sphere.

Kowalski and Rembieliński do not have a parameter comparable to our $\omega$; the only dimensional parameters in 18] are $m, r$, and $\hbar$. This affects the interpretation of their Eq. (6.1) for the complex coordinates on phase space (what we call a). Equation (6.1) involves $\cosh l$ and $\sinh l$, where $l$ is the classical angular momentum. The argument of cosh and sinh should be dimensionless, and the only way to make $l$ dimensionless using only $m, r$, and $\hbar$ is to divide $l$ by $\hbar$. Thus in Eq. (6.1) of 18 $l$ implicitly means $l / \hbar$. In our view it is unnatural in a classical formula to insist that the angular momentum be measured in units of Planck's constant. In our approach (see (18) below), angular momentum is measured in units of $m \omega r^{2}$. Although nothing prevents one from choosing $\omega$ so that $m \omega r^{2}=\hbar$, it seems artificial to us to insist on this. After all, Eq. (6.1) concerns a classical construction that ought to be independent of the value of Planck's constant.

We are now ready to apply the "complexifier" method of Thiemann [7]. We take as our classical complexifier the kinetic energy function divided by $\omega$,

$$
\text { complexifier }=\frac{\text { kinetic energy }}{\omega}=\frac{j^{2}}{2 m \omega r^{2}} .
$$

We then define complex-valued functions $a_{1}, \cdots, a_{d+1}$ on $T^{*}\left(S^{d}\right)$ by

$$
\begin{aligned}
a_{k}(\mathbf{x}, \mathbf{p}) & =\exp \left(i\left\{\cdot \frac{j^{2}}{2 m \omega r^{2}}\right\}\right) x_{k} \\
& =\sum_{n=0}^{\infty}\left(\frac{i}{2 m \omega r^{2}}\right)^{n} \frac{1}{n !} \underbrace{\left\{\cdots\left\{\left\{x_{k}, j^{2}\right\}, j^{2}\right\}, \cdots, j^{2}\right\}}_{n} .
\end{aligned}
$$

Note that the $a_{k}$ 's are obtained from the $x_{k}$ 's by means of the classical timeevolution generated by the kinetic energy function, evaluated at the imaginary time $i / \omega$. The calculations below will show that the series (15) converges for all $\mathbf{x}$ and $\mathbf{p .}$

In [7] Thiemann allows any function $C$ on the phase space to be the complexifier, provided that $\exp (i\{\cdot, C\}) x_{k}$ converges. (Thiemann also allows any cotangent 
bundle to be the phase space.) The condition of convergence, however, imposes severe restrictions on the choice of $C$, even when $C$ is quadratic in the momenta. We consider in this paper only the complexifier (14).

To compute the functions $a_{k}$ explicitly, we first compute using (11) and (13) that, in vector notation,

$$
\left\{\mathbf{x}, \frac{j^{2}}{2 m \omega r^{2}}\right\}=\frac{1}{m \omega r^{2}} \mathbf{j} \mathbf{x}=\frac{1}{m \omega} \mathbf{p} .
$$

On the other hand, it is easily verified that $\left\{j_{k l}, j^{2}\right\}=0$, which means that if we compute further Poisson brackets with $j^{2}$, the matrix $\mathbf{j}$ gets ignored and we get

$$
\left(\frac{1}{2 m \omega r^{2}}\right)^{n} \underbrace{\left\{\cdots\left\{\left\{\mathbf{x}, j^{2}\right\}, j^{2}\right\}, \cdots, j^{2}\right\}}_{n}=\left(\frac{1}{m \omega r^{2}}\right)^{n} \mathbf{j}^{n} \mathbf{x} .
$$

Here $\mathbf{j}^{n} \mathbf{x}$ means the matrix $\mathbf{j}$ applied $n$ times to the vector $\mathbf{x}$.

We obtain, then, the following "polar coordinates" expression for $\mathbf{a}=\left(a_{1}, \cdots, a_{d+1}\right)$

$$
\mathbf{a}(\mathbf{x}, \mathbf{p})=e^{i \mathbf{j}(x, p) / m \omega r^{2}} \mathbf{x} .
$$

(Compare Eq. (3.37) in the first entry of [8].) Using (10) we can compute this explicitly as

$$
\begin{aligned}
\mathbf{a}(\mathbf{x}, \mathbf{p}) & =\cosh \left(\frac{p}{m \omega r}\right) \mathbf{x}+i \frac{r}{p} \sinh \left(\frac{p}{m \omega r}\right) \mathbf{p} \\
& =\cosh \left(\frac{j}{m \omega r^{2}}\right) \mathbf{x}+i \frac{r^{2}}{j} \sinh \left(\frac{j}{m \omega r^{2}}\right) \mathbf{p} .
\end{aligned}
$$

We may at this point check the units: $p / m \omega r=j / m \omega r^{2}$ is dimensionless and the whole expression has units of position. Note also that $\mathbf{a}(\mathbf{x},-\mathbf{p})=\overline{\mathbf{a}(\mathbf{x}, \mathbf{p})}$.

With $d=2$ (and $r=m \omega=1$ ) (18) agrees with Eq. (6.1) of [18]. (See also Eq. (3.6) in the second entry of [8].) In any dimension (18) agrees with the "adapted complex structure" on $T^{*}\left(S^{d}\right)$ as defined by Lempert and Szőke [21] and Guillemin and Stenzel 222, which for the special case of rank one symmetric spaces was constructed earlier by Morimoto and Nagano [23]. See for example p. 410 of 24].

It is instructive to consider how this works out in the case of $S^{1} \subset \mathbb{R}^{2}$. In that case we have only a single component of angular momentum, $j_{12}=p_{1} x_{2}-p_{2} x_{1}$, so that $j=\left|j_{12}\right|$. Since both terms in (18) are even functions of $j$, we may replace $j$ by $j_{12}$ there. Then let $\theta$ be the usual angular coordinate and let $\rho=-j_{12} / m \omega r^{2}$, so that $\rho$ is (up to a constant) the canonically conjugate momentum to $\theta$. Our phase space is the set of points $\left(x_{1}, x_{2}, p_{1}, p_{2}\right)$ with $x_{1}^{2}+x_{2}^{2}=r^{2}$ and $p_{1} x_{1}+p_{2} x_{2}=0$. On this set we have the easily verified identity $j_{12}\left(x_{2},-x_{1}\right)=r^{2}\left(p_{1}, p_{2}\right)$. Upon using this identity and $\mathbf{x}=r(\cos \theta, \sin \theta),(18)$ becomes

$$
\begin{aligned}
\mathbf{a} & =r(\cosh \rho \cos \theta-i \sinh \rho \sin \theta, \cosh \rho \sin \theta+i \sinh \rho \cos \theta) \\
& =r(\cos (\theta+i \rho), \sin (\theta+i \rho))
\end{aligned}
$$

This result facilitates comparison with the analysis of the $S^{1}$ case in [19] and should be thought of as the "complexification" of the identity $\mathbf{x}=r(\cos \theta, \sin \theta)$.

As is well known, the Poisson bracket satisfies a Leibniz-type product rule, $\left\{f_{1}, f_{2} f_{3}\right\}=\left\{f_{1}, f_{2}\right\} f_{3}+f_{2}\left\{f_{1}, f_{3}\right\}$, and the analogous formula for Poisson brackets, $\left\{f_{1},\left\{f_{2}, f_{3}\right\}\right\}=\left\{\left\{f_{1}, f_{2}\right\}, f_{3}\right\}+\left\{f_{2},\left\{f_{1}, f_{3}\right\}\right\}$. (This last expression is equivalent to the Jacobi identity.) Suppose then that we define the "complexification" $f_{\mathbb{C}}$ 
of any function $f$ to be

$$
f_{\mathbb{C}}=e^{i\{\cdot, \text { complexifier }\}} f
$$

whenever the power series for the exponential converges. Then by a standard power series argument we have

$$
\left(f_{1} f_{2}\right)_{\mathbb{C}}=\left(f_{1}\right)_{\mathbb{C}}\left(f_{2}\right)_{\mathbb{C}}
$$

and

$$
\left\{f_{1}, f_{2}\right\}_{\mathbb{C}}=\left\{\left(f_{1}\right)_{\mathbb{C}},\left(f_{2}\right)_{\mathbb{C}}\right\}
$$

Equation (20) shows that if we "complexify" any polynomial in the variables $x_{1}, \cdots, x_{d+1}$ we will get simply the same polynomial in $a_{1}, \cdots, a_{d+1}$. Furthermore, since $\left\{x_{k}, x_{l}\right\}=$ 0 , (21) shows that

$$
\left\{a_{k}, a_{l}\right\}=0,
$$

which implies that $\left\{\bar{a}_{k}, \bar{a}_{l}\right\}=0$. The formula for $\left\{a_{k}, \bar{a}_{l}\right\}$, however, is complicated and we will not compute it here.

Equation (20) also shows that

$$
a^{2}(\mathbf{x}, \mathbf{p})=r^{2}
$$

for all $\mathbf{x}, \mathbf{p}$, which is also evident from (18). Thus the map $(\mathbf{x}, \mathbf{p}) \rightarrow \mathbf{a}(\mathbf{x}, \mathbf{p})$ defines a map of the cotangent bundle $T^{*}\left(S^{d}\right)$ to the complexified sphere

$$
S_{\mathbb{C}}^{d}=\left\{\mathbf{a} \in \mathbb{C}^{d+1} \mid a_{1}^{2}+\cdots+a_{d+1}^{2}=r^{2}\right\} .
$$

It is not hard to see that this map is invertible, indeed a diffeomorphism of $T^{*}\left(S^{d}\right)$ with $S_{\mathbb{C}}^{d}$.

\section{The AnNihilation Operators}

We now consider the quantum counterpart of the constructions in the previous section. This means that the functions $j_{k l}$ and $x_{k}$ should be replaced by selfadjoint operators $J_{k l}$ and $X_{k}$ acting on (suitable domains in) some separable complex Hilbert space. These should satisfy $J_{l k}=-J_{k l}$ and the quantum counterpart of the Poisson-bracket relations (11), namely,

$$
\begin{aligned}
\frac{1}{i \hbar}\left[J_{k l}, J_{m n}\right] & =\delta_{k n} J_{l m}+\delta_{l m} J_{k n}-\delta_{k m} J_{l n}-\delta_{l n} J_{k m} \\
\frac{1}{i \hbar}\left[X_{k}, J_{l m}\right] & =\delta_{k l} X_{m}-\delta_{k m} X_{l} \\
\frac{1}{i \hbar}\left[X_{k}, X_{l}\right] & =0
\end{aligned}
$$

We recognize this as a representation of the Euclidean Lie algebra e $(d+1)=$ $\operatorname{so}(d+1) \ltimes \mathbb{R}^{d+1}$. We assume that this representation of $\mathrm{e}(d+1)$ comes from an irreducible unitary representation of the associated connected, simply connected Lie group $\tilde{\mathrm{E}}(d+1)$. Here $\tilde{\mathrm{E}}(d+1) \cong \operatorname{Spin}(d+1) \ltimes \mathbb{R}^{d+1}$ for $d \geq 2$ and $\tilde{\mathrm{E}}(2) \cong \mathbb{R} \ltimes \mathbb{R}^{2}$, where $\ltimes$ denotes a semidirect product with the normal factor on the right.

The irreducible unitary representations of $\tilde{\mathrm{E}}(d+1)$ may be classified by the Wigner-Mackey method. One first chooses an orbit of $\operatorname{Spin}(d+1)$ on $\mathbb{R}^{d+1}$, namely, a sphere of some radius $r \geq 0$. Since the case $r=0$ is presumably unphysical (though mathematically permitted), we assume from now on that $r>0$. Next one selects 
any one point in the sphere of radius $r$ and considers the "little group," that is, the stabilizer in $\operatorname{Spin}(d+1)$ of the point. For $r>0$ the little group is simply $\operatorname{Spin}(d)$. The irreducible representations of $\tilde{\mathrm{E}}(d+1)$ are then labeled by the value of $r$ and by an irreducible unitary representation of the little group. In this paper we will consider only the case in which the representation of the little group is trivial. Nevertheless the definitions of the annihilation operators and of the coherent states make sense in general.

Choosing a sphere of radius $r$ amounts to requiring that the operators in 25) satisfy

$$
X^{2}=r^{2}
$$

We will shortly impose an additional condition among the $X$ 's and $J$ 's that forces the representation of the little group to be trivial. For now, however, we will assume only the e $(d+1)$ relations (25) and the condition (26).

We define the total angular momentum $J^{2}$ as in the classical case by

$$
J^{2}=\sum_{k<l} J_{k l}^{2}
$$

As in the classical case we define

$$
\text { complexifier }=\frac{\text { kinetic energy }}{\omega}=\frac{J^{2}}{2 m \omega r^{2}} .
$$

We then define the annihilation operators by replacing $\{\cdot$, complexifier $\}$ in (15) with its quantum counterpart, $(1 / i \hbar)[\cdot$, complexifier $]$ :

$$
\begin{aligned}
A_{k} & =\exp \left(\frac{i}{i \hbar}\left[\cdot, \frac{J^{2}}{2 m \omega r^{2}}\right]\right) X_{k} \\
& =\sum_{n=0}^{\infty} \frac{1}{\left(2 m \omega r^{2} \hbar\right)^{n}} \frac{1}{n !}\left[\cdots\left[\left[X_{k}, J^{2}\right], J^{2}\right] \cdots, J^{2}\right] .
\end{aligned}
$$

By a standard formula from Lie group theory this may be written as

$$
A_{k}=e^{-J^{2} / 2 m \omega r^{2} \hbar} X_{k} e^{J^{2} / 2 m \omega r^{2} \hbar}
$$

In the general form of Thiemann's method, (29) would be $\exp (-\hat{C} / \hbar) X_{k} \exp (\hat{C} / \hbar)$, where $\hat{C}$ is the quantum operator corresponding to the classical complexifier $C$.

For determining the eigenvectors of the annihilation operators (i.e. the coherent states), (29) is the most useful expression for the $A_{k}$ 's. Nevertheless we will give two other formulas, a polar decomposition and an "explicit" formula in terms of the position and momentum operators. The $A_{k}$ 's are unbounded operators and so something must be said about their domains; see the discussion at the end of this section. The annihilation operators satisfy (in analogy to (22) and (23))

$$
\begin{aligned}
{\left[A_{k}, A_{l}\right] } & =0 \\
A^{2} & =r^{2}
\end{aligned}
$$

(since $\left[X_{k}, X_{l}\right]=0$ and $\left.X^{2}=r^{2}\right)$. 
To compute $\mathbf{A}$ we first compute using (25) and (27) that

$$
\begin{aligned}
\frac{1}{i \hbar}\left[X_{k}, J^{2}\right] & =J_{k l} X_{l}+X_{l} J_{k l} \\
& =2 J_{k l} X_{l}+i \hbar\left(\delta_{l k} X_{l}-\delta_{l l} X_{k}\right) \\
& =2 J_{k l} X_{l}-i \hbar d X_{k} .
\end{aligned}
$$

Here we have chosen to order things with the $J$ 's to the left of the $X$ 's and we use the sum convention. Thus in vector notation we have

$$
\frac{1}{i \hbar}\left[\mathbf{X}, \frac{J^{2}}{2 m \omega r^{2}}\right]=\frac{1}{m \omega r^{2}}\left(\mathbf{J}-\frac{i \hbar d}{2}\right) \mathbf{X} .
$$

Here the term involving $i \hbar d / 2$ is a "quantum correction"; compare (16).

Since $\left[J_{k l}, J^{2}\right]=0$, further brackets will give just another factor of the matrix operator $\mathbf{J}-i \hbar d / 2$. Thus the polar coordinates decomposition of $\mathbf{A}$ has just a single quantum correction, namely,

$$
\mathbf{A}=\exp \left\{\frac{i \mathbf{J}+\hbar d / 2}{m \omega r^{2}}\right\} \mathbf{X}
$$

In the case $d=2$, a formula very similar to this is taken in [18] as the definition of the annihilation operators. The only difference is that Kowalski and Rembieliński formulate things in terms of $2 \times 2$ matrix operators, whereas in the case $d=2(33)$ is in terms of $3 \times 3$ matrix operators. Nevertheless, our expression is equivalent to that of Kowalski and Rembieliński; see below and Eq. (5.9) of [20]. The analog of (33) for the group case is given in Eq. (3.44) of the first entry in 8] and in Eq. (3.13) of the second entry in [8].

Note that the definition (28) makes sense in any irreducible representation of $\tilde{\mathrm{E}}(d+1)$ (with $X^{2}=r^{2}>0$ ), and that the formula (33) is valid in this generality. However, to compute $\mathbf{A}$ more explicitly than this we need to further specify the irreducible representation of $\tilde{\mathrm{E}}(d+1)$. We limit ourselves to the case in which the representation of the little group $\operatorname{Spin}(d)$ is trivial. This corresponds to a quantum particle on the sphere with no internal degrees of freedom. In the case the case of $S^{2}$, this corresponds to taking the "twist" (in the notation of Kowalski and Rembieliński) to be zero. We show in Section 9 that the little group acts trivially if and only if the following relation holds:

$$
X^{2} J_{k l}=J_{k m} X_{m} X_{l}-J_{l m} X_{m} X_{k} .
$$

This is the quantum counterpart of the classical constraint (9). by

In computing $\mathbf{A}$ it is convenient to introduce "momentum" operators $P_{k}$ given

$$
P_{k}:=\frac{J_{k l} X_{l}}{r^{2}} .
$$

These operators are not self-adjoint and we have chosen to put the $J$ 's to the left of the $X$ 's (because we have put $\mathbf{J}$ to the left of $\mathbf{X}$ in (32) and (33)). We may re-write (34) in terms of the $P_{k}$ 's as

$$
J_{k l}=P_{k} X_{l}-P_{l} X_{k} .
$$

The position and momentum operators satisfy

$$
\frac{1}{i \hbar}\left[X_{k}, P_{l}\right]=\delta_{k l} I-\frac{X_{k} X_{l}}{r^{2}}
$$


(Compare (12).) We may also compute using (25) the quantum counterpart of $\mathbf{x} \cdot \mathbf{p}=0$, which is really two relations on the quantum side:

$$
\begin{aligned}
& \mathbf{P} \cdot \mathbf{X}=0 \\
& \mathbf{X} \cdot \mathbf{P}=i \hbar d I .
\end{aligned}
$$

We now write down the formulas that allow us to compute $\mathbf{A}$ in terms of $\mathbf{X}$ and P:

$$
\begin{aligned}
& \mathbf{J X}=r^{2} \mathbf{P} \\
& \mathbf{J P}=-P^{2} \mathbf{X}+i \hbar(d-1) \mathbf{P} .
\end{aligned}
$$

The first line is simply the definition of $\mathbf{P}$. The second line comes from (34) or (35) and is essential to the explicit calculation of the annihilation operators in terms of $\mathbf{X}$ and $\mathbf{P}$. Note that there is an additional quantum correction here. To verify the second line of (37), write $\mathbf{J}$ in terms of $\mathbf{P}$ using (35) and then use (36).

We now treat $\mathbf{J}$ as a $2 \times 2$ matrix acting on the "basis" $\mathbf{X}$ and $\mathbf{P}$, as given in (37). Since all the entries of this $2 \times 2$ matrix commute, we can just treat $P^{2}$ as a scalar and compute an ordinary $2 \times 2$ matrix exponential. So effectively we have

$$
\mathbf{J}=\left(\begin{array}{cc}
0 & -P^{2} \\
r^{2} & i \hbar(d-1)
\end{array}\right)
$$

One can then compute the exponential of this matrix either by hand or using a computer algebra program. A calculation shows that $P^{2}=r^{-2} J^{2}$ as in the classical case. It is convenient to express things in terms of the scalar operator

$$
J:=\sqrt{J^{2}+\hbar^{2}(d-1)^{2} / 4} .
$$

Then after exponentiating $\mathbf{J}$, (33) becomes

$$
\begin{aligned}
\mathbf{A} & =e^{\hbar / 2 m \omega r^{2}} \cosh \left(\frac{J}{m \omega r^{2}}\right) \mathbf{X}+e^{\hbar / 2 m \omega r^{2}} \frac{\hbar(d-1)}{2 J} \sinh \left(\frac{J}{m \omega r^{2}}\right) \mathbf{X} \\
& +i e^{\hbar / 2 m \omega r^{2}} \frac{r^{2}}{J} \sinh \left(\frac{J}{m \omega r^{2}}\right) \mathbf{P} .
\end{aligned}
$$

Equation (38) is similar to the corresponding classical expression (18), with only the following differences: 1) there is an overall factor of $\left.\exp \left(\hbar / 2 m \omega r^{2}\right), 2\right)$ the quantity $j$ in $(18)$ is replaced by $\left(J^{2}+\hbar^{2}(d-1)^{2} / 4\right)^{1 / 2}$, and 3) there is an extra sinh term in the coefficient of $\mathbf{X}$ that does not occur in the classical formula. Note that the above expression formally coincides with the classical one in the limit $\hbar \rightarrow 0$. In the case $d=2$ with $r=m \omega=\hbar=1$ (38) agrees with Eq. (4.16) in [18]. In the case $d=3$ (identifying $S^{3}$ with $\mathrm{SU}(2)$ and adjusting for minor differences of normalization) (38) agrees with Eq. (3.132) in the last entry in [8]. In the case $d=1$ we get an expression identical to the classical expression (19) except for an overall factor of $\exp \left(\hbar / m \omega r^{2}\right)$ (compare Eqs. (3.3) and (3.4) of [19]).

It is clear from (38) that the $A_{k}$ 's are unbounded operators, as expected since the $a_{k}$ 's are unbounded functions. This means that the $A_{k}$ 's cannot be defined on the whole Hilbert space, but only on some dense subspace, which should be specified. We take the expression (29) as our definition of the annihilation operators. We first define the $A_{k}$ 's on what we will call the "minimal domain," namely, the space of finite linear combinations of spherical harmonics (that is, of eigenvectors for $J^{2}$ ). The expression (29) makes sense on the minimal domain, since each of the three factors making up $A_{k}$ preserve this space. We consider also a "maximal domain" 
for the $A_{k}$ 's, defined as follows. Given any vector $|\phi\rangle$ in the Hilbert space, we expand $|\phi\rangle$ in a series expansion in terms of spherical harmonics. Then we apply $A_{k}$ term-by-term, that is, by formally interchanging $A_{k}$ with the sum. The result will then be a formal series of spherical harmonics. If this formal series converges in the Hilbert space then we say that $|\phi\rangle$ is in the maximal domain of $A_{k}$ and that the value of $A_{k}|\phi\rangle$ is the sum of this series. (It can be shown that the product of $x_{k}$ and a spherical harmonic of degree $n$ is the sum of a spherical harmonic of degree $n+1$ and a spherical harmonic of degree $n-1$. It follows that the degree $l$ term in the expansion of $A_{k}|\phi\rangle$ involves only the degree $n-1$ and degree $n+1$ terms of $|\phi\rangle$. So each term in the formal series for $A_{k}|\phi\rangle$ can be computed by means of a finite sum.)

It can be shown that if one starts with the operator $A_{k}$ on its minimal domain and then takes its closure (in the functional analytic sense) the result is the operator $A_{k}$ on its maximal domain. Thus if we want $A_{k}$ to be a closed operator there is only one reasonable choice for its domain. The coherent states will not be finite linear combinations of spherical harmonics but will be in the maximal domain of all the $A_{k}$ 's.

\section{The COHEREnt STATES}

We are now ready to introduce the coherent states, which we define to be the simultaneous eigenvectors of the annihilation operators. These coherent states are not of Perelomov type. Although we have described the quantum Hilbert space as an irreducible representation of $\tilde{\mathrm{E}}(d+1)$, the coherent states are not obtained from one fixed vector by the action of $\tilde{\mathrm{E}}(d+1)$. Indeed the only elements of $\tilde{\mathrm{E}}(d+1)$ that preserve the set of coherent states are the rotations. See Section 10 for a comparison of these coherent states to the generalized Perelomov-type coherent states for $\tilde{\mathrm{E}}(d+1)$, as constructed either by De Bièvre or by Isham and Klauder.

The coherent states will be simultaneous eigenvectors of the annihilation operators $A_{k}$, and thus can be thought of as the quantum counterparts of a classical state with definite values for the complex coordinates $a_{k}$. On the quantum side, however, $A_{k}^{\dagger}$ does not commute with $A_{k}$, and thus although the coherent states satisfy $A_{k}|\psi\rangle=a_{k}|\psi\rangle$ they do not satisfy $A_{k}^{\dagger}|\psi\rangle=\bar{a}_{k}|\psi\rangle$.

We use the formula (29) for the annihilation operators. If we introduce the dimensionless form of the total angular momentum,

$$
\tilde{J}^{2}=\frac{1}{\hbar^{2}} J^{2}
$$

then this may be expressed as

$$
\mathbf{A}=e^{-\tau \tilde{J}^{2} / 2} \mathbf{X} e^{\tau \tilde{J}^{2} / 2},
$$

where $\tau$ is the dimensionless quantity given by

$$
\tau=\frac{\hbar}{m \omega r^{2}} .
$$

The parameter $\tau$ is a new feature of the sphere case; no such dimensionless quantity arises in the $\mathbb{R}^{d}$ case. The significance of $\tau$ for the coherent states is that it controls the ratio of the spatial width of the coherent states to the radius of the sphere. Specifically, we expect the approximate spatial width $\Delta X$ of a coherent 
state to be $\sqrt{\hbar / 2 m \omega}$, at least if this quantity is small compared to $r$. In that case

$$
\frac{\Delta X}{r} \approx \frac{\sqrt{\hbar / 2 m \omega}}{r}=\sqrt{\frac{\tau}{2}}
$$

So if $\tau \ll 1$ we expect the coherent states to be concentrated in a small portion of the sphere, and to look, in appropriate coordinates, approximately Gaussian. This has been proved [8] for the case of $S^{3}=\mathrm{SU}(2)$.

Kowalski and Rembieliński implicitly take $\tau=1$ in their treatment of the $d=2$ case, since they choose units with $m=r=\hbar=1$, and since they do not have the parameter $\omega$. (See our comments in Section 3 about the parameter $\omega$.) To us this seems a needless loss of generality, even though it is easy to insert $\tau$ in the appropriate places in their formulas.

We now proceed with the construction of the coherent states. For each a in the real sphere $S^{d}$, let $\left|\delta_{\mathbf{a}}\right\rangle$ be the (generalized) position eigenfunction with $X_{k}\left|\delta_{\mathbf{a}}\right\rangle=a_{k}\left|\delta_{\mathbf{a}}\right\rangle$. Since we assume that the little group acts trivially these position eigenfunctions are (for each $\mathbf{a}$ ) unique up to a constant and we may normalize them so that the action of the rotation group takes $\left|\delta_{\mathbf{a}}\right\rangle$ to $\left|\delta_{R \mathbf{a}}\right\rangle, R \in \mathrm{SO}(d+1)$. If we let

$$
\left|\psi_{\mathbf{a}}\right\rangle=e^{-\tau \tilde{J}^{2} / 2}\left|\delta_{\mathbf{a}}\right\rangle
$$

then it follows immediately from (39) that $\left|\psi_{\mathbf{a}}\right\rangle$ is a simultaneous eigenvector for each $A_{k}$ with eigenvalue $a_{k}$. Although $\left|\delta_{\mathbf{a}}\right\rangle$ is non-normalizable, the smoothing nature of the operator $\exp \left(-\tau \tilde{J}^{2} / 2\right)$ guarantees that $\left|\psi_{\mathbf{a}}\right\rangle$ is normalizable for all $\mathbf{a} \in S^{d}$. A key result of this section is the following proposition, which asserts that we can analytically continue the coherent states $\left|\psi_{\mathbf{a}}\right\rangle$ with respect to a so as to obtain states labeled by points a in the complex sphere $S_{\mathbb{C}}^{d}$.

Proposition 1. There exists a unique family of states $\left|\psi_{\mathbf{a}}\right\rangle$ parameterized by $\mathbf{a} \in$ $S_{\mathbb{C}}^{d}$ such that 1) the states depend holomorphically on $\mathbf{a}$, and 2) for $\mathbf{a} \in S^{d}$, they agree with the states in (40). These are normalizable states and satisfy

$$
A_{k}\left|\psi_{\mathbf{a}}\right\rangle=a_{k}\left|\psi_{\mathbf{a}}\right\rangle, \quad \mathbf{a} \in S_{\mathbb{C}}^{d}
$$

We call these states the coherent states. Note that we have then one coherent state for each point in $S_{\mathbb{C}}^{d}$, that is, one coherent state for each point in the classical phase space. It can be shown that these are (up to a constant) the only simultaneous eigenvectors of the annihilation operators. These coherent states are not normalized to be unit vectors. The proof of Proposition 1 is at the end of this section.

Note that since the operator $\tilde{J}^{2}$ commutes with rotations, the action of the rotation subgroup $\mathrm{SO}(d+1)$ of $\mathrm{E}(d+1)$ will take $\left|\psi_{\mathbf{a}}\right\rangle$ to $\left|\psi_{R \mathbf{a}}\right\rangle$ for any $R \in$ $\mathrm{SO}(d+1)$. On sufficiently regular states we can analytically continue the action of $\mathrm{SO}(d+1)$ to an action of $\mathrm{SO}(d+1 ; \mathbb{C})$, which will take $\left|\psi_{\mathbf{a}}\right\rangle$ to $\left|\psi_{R \mathbf{a}}\right\rangle$ for any $R \in \mathrm{SO}(d+1 ; \mathbb{C})$. Then any coherent state can be obtained from any other by the action of $\mathrm{SO}(d+1 ; \mathbb{C})$. Since, however, the action of $\mathrm{SO}(d+1 ; \mathbb{C})$ is neither unitary nor irreducible, this observation still does not bring the coherent states into the Perelomov framework.

We can give an explicit formula for the coherent states in the position representation in terms of the heat kernel on $S^{d}$. The heat kernel is the function on $S^{d} \times S^{d}$ given by $\rho_{\tau}(\mathbf{x}, \mathbf{y})=\left\langle\delta_{\mathbf{x}}\left|e^{-\tau \tilde{J}^{2} / 2}\right| \delta_{\mathbf{y}}\right\rangle$. It can be shown (see [1] or the formulas below) the $\rho_{\tau}$ extends (uniquely) to a holomorphic function on $S_{\mathbb{C}}^{d} \times S_{\mathbb{C}}^{d}$, also denoted 
$\rho_{\tau}$. In terms of the analytically continued heat kernel the coherent states are given by

$$
\left\langle\delta_{\mathbf{x}} \mid \psi_{\mathbf{a}}\right\rangle=\rho_{\tau}(\mathbf{a}, \mathbf{x}), \quad \mathbf{a} \in S_{\mathbb{C}}^{d}, \mathbf{x} \in S^{d} .
$$

Meanwhile, explicit formulas for the heat kernel may be found, for example, in [25, 26]. For $\mathbf{x}$ and $\mathbf{y}$ in the real sphere, $\rho_{\tau}(\mathbf{x}, \mathbf{y})$ depends only on the angle $\theta$ between $\mathbf{x}$ and $\mathbf{y}$, where $\theta=\cos ^{-1}\left(\mathbf{x} \cdot \mathbf{y} / r^{2}\right)$. This remains true for $\rho_{\tau}(\mathbf{a}, \mathbf{x})$, with $\mathbf{a} \in S_{\mathbb{C}}^{d}$, except now $\theta=\cos ^{-1}\left(\mathbf{a} \cdot \mathbf{x} / r^{2}\right)$ is complex-valued. Of course the inverse cosine function is multiple-valued, but because the heat kernel is an even, $2 \pi$-periodic function of $\theta$, it does not matter which value of $\theta$ we use, provided that $\cos \theta=\mathbf{a} \cdot \mathbf{x} / r^{2}$.

We now record the formulas, writing $\rho_{\tau}^{d}$ to indicate the dependence on the dimension. For $d=1,2,3$ we have

$$
\begin{aligned}
& \rho_{\tau}^{1}(\mathbf{a}, \mathbf{x})=(2 \pi \tau)^{-1 / 2} \sum_{n=-\infty}^{\infty} e^{-(\theta-2 \pi n)^{2} / 2 \tau} \\
& \rho_{\tau}^{2}(\mathbf{a}, \mathbf{x})=(2 \pi \tau)^{-1} e^{\tau / 8} \frac{1}{\sqrt{\pi \tau}} \int_{\theta}^{\pi} \frac{1}{\sqrt{\cos \theta-\cos \phi}} \sum_{n=-\infty}^{\infty}(-1)^{n}(\phi-2 \pi n) e^{-(\phi-2 \pi n)^{2} / 2 \tau} d \phi \\
& \rho_{\tau}^{3}(\mathbf{a}, \mathbf{x})=(2 \pi \tau)^{-3 / 2} e^{\tau / 2} \frac{1}{\sin \theta} \sum_{n=-\infty}^{\infty}(\theta-2 \pi n) e^{-(\theta-2 \pi n)^{2} / 2 \tau}
\end{aligned}
$$

In the formula for $\rho_{\tau}^{2}$ we may without loss of generality take $\theta$ with $0 \leq \operatorname{Re} \theta \leq \pi$, in which case the integral is to be interpreted as a contour integral in the strip $0 \leq \operatorname{Re} \phi \leq \pi$. The relatively simple formula for the heat kernel on $S^{3}=\operatorname{SU}(2)$ allows for detailed calculations for the coherent states in this case, as carried out in [8]. To find the formula in higher dimensions we use the inductive formula

$$
\rho_{\tau}^{d+2}(\mathbf{a}, \mathbf{x})=-e^{d \tau / 2} \frac{1}{2 \pi \sin \theta} \frac{d}{d \theta} \rho_{\tau}^{d}(\mathbf{a}, \mathbf{x}) .
$$

There is also an expression for the heat kernel in terms of spherical harmonics. For example, when $d=2$ we have

$$
\rho_{\tau}^{2}(\mathbf{a}, \mathbf{x})=\sum_{l=0}^{\infty} e^{-\tau l(l+1) / 2} \sqrt{2 l+1} P_{l}(\cos \theta),
$$

where the $P_{l}$ is the Legendre polynomial of degree $l$. (Compare Eq. (5.3) of 18 .) The earlier expression for $\rho_{\tau}^{2}$ is a sort of Poisson-summed version of (42)-see [26].

We also consider the reproducing kernel, defined by

$$
R_{\tau}(\mathbf{a}, \mathbf{b})=\left\langle\psi_{\mathbf{b}} \mid \psi_{\mathbf{a}}\right\rangle, \quad \mathbf{a}, \mathbf{b} \in S_{\mathbb{C}}^{d}
$$

In terms of the analytically continued heat kernel the reproducing kernel is given by

$$
R_{\tau}(\mathbf{a}, \mathbf{b})=\rho_{2 \tau}(\mathbf{a}, \overline{\mathbf{b}}), \quad \mathbf{a}, \mathbf{b} \in S_{\mathbb{C}}^{d} .
$$

Note that $R_{\tau}(\mathbf{a}, \mathbf{b})$ depends holomorphically on $\mathbf{a}$ and anti-holomorphically on $\mathbf{b}$.

In the case of $S^{1}=\mathrm{U}(1)$ and $S^{3}=\mathrm{SU}(2)$, Thiemann and Winkler have proved in the second and third entries of [8] that the coherent states defined here satisfy good phase space localization properties and that the Ehrenfest theorem holds infinitesimally. We fully expect that these results hold for all $d$. This expectation is based on the idea that the heat kernel in (41) will behave for small $\tau$ like $c \exp \left(-\theta^{2} / 2 \tau\right)$, 
even for complex values of $\theta$. Thiemann and Winkler have verified this in the cases $d=1,3$ and a similar analysis should be possible in general, using the explicit formulas for small $d$ and the inductive formula for $\rho^{d+2}$ in terms of $\rho^{d}$.

Proof of Proposition [1. There are two ways to prove this proposition. The simplest way is to use the expression for $\psi_{\mathbf{a}}$ in terms of the heat kernel $\rho_{\tau}$ and the explicit formulas above for $\rho_{\tau}$. It is easily seen that $\rho_{\tau}$ extends to an entire holomorphic function of $\theta$. Thus the expression $\left\langle\delta_{\mathbf{x}} \mid \psi_{\mathbf{a}}\right\rangle=\rho_{\tau}(\mathbf{a}, \mathbf{x})$ makes sense for any $\mathbf{a}$ in $S_{\mathbb{C}}^{d}$, with $\cos \theta$ and thus also $\theta$ taking complex values. It is not hard to see that the $\left|\psi_{\mathbf{a}}\right\rangle$, so defined, is in the (maximal) domain of the annihilation operators and that it depends holomorphically on $\mathbf{a} \in S_{\mathbb{C}}^{d}$. Since $A_{k}\left|\psi_{\mathbf{a}}\right\rangle=a_{k}\left|\psi_{\mathbf{a}}\right\rangle$ for $\mathbf{a} \in S^{d}$, an analytic continuation argument will show that this equation remains true for all $\mathbf{a} \in S_{\mathbb{C}}^{d}$. Alternatively we may use the expansion of the coherent states in terms of spherical harmonics as in (42) and show that this expression can be analytically continued term-by-term in a. (Compare Section 4 of [i].)

\section{The Resolution of THE IDENTITY}

We now choose a coordinate system in which $r=1$ and $m \omega=1$. This amounts to using the normalized position $\mathbf{x} / r$ and normalized momentum $\mathbf{p} / m \omega r$. Since these choices set our position and momentum scales we cannot also take $\hbar=1$. Note that the dimensionless parameter $\tau=\hbar / m \omega r^{2}$ equals $\hbar$ in such a coordinate system. We now write $\left|\psi_{\mathbf{x}, \mathbf{p}}\right\rangle$ for $\left|\psi_{\mathbf{a}(\mathbf{x}, \mathbf{p})}\right\rangle$.

Theorem 2. The coherent states have a resolution of the identity of the form

$$
I=\int_{\mathbf{x} \in S^{d}} \int_{\mathbf{p} \cdot \mathbf{x}=0}\left|\psi_{\mathbf{x}, \mathbf{p}}\right\rangle\left\langle\psi_{\mathbf{x}, \mathbf{p}}\right| \nu(2 \tau, 2 p)\left(\frac{\sinh 2 p}{2 p}\right)^{d-1} 2^{d} d \mathbf{p} d \mathbf{x}
$$

where $\nu(s, R)$ is the solution to the differential equation

$$
\frac{d \nu(s, R)}{d s}=\frac{1}{2}\left[\frac{\partial^{2} \nu}{\partial R^{2}}-(d-1) \frac{\cosh R}{\sinh R} \frac{\partial \nu}{\partial R}\right]
$$

subject to the initial condition

$$
\lim _{s \downarrow 0} c_{d} \int_{0}^{\infty} f(R) \nu(s, R)(\sinh R)^{d-1} d R=f(0)
$$

for all continuous functions $f$ on $[0, \infty)$ with at most exponential growth at infinity. Here $d \mathbf{x}$ is the surface area measure on $S^{d}, \tau$ is the dimensionless quantity $\tau=$ $\hbar / m \omega r^{2}$, and $c_{d}$ is the volume of the unit sphere in $\mathbb{R}^{d}$.

The operator on the right side of the equation for $\nu$ is just the radial part of the Laplacian for $d$-dimensional hyperbolic space [27, Sect. 5.7]. This means that $\nu(s, R)$ is the heat kernel for hyperbolic space, that is, the fundamental solution of the heat equation. Hyperbolic space is the non-compact, negatively curved "dual" of the compact, positively curved symmetric space $S^{d}$. Note that the function $\nu$ is evaluated at "time" $2 \tau$ and radius $2 p$. The inversion formula for the SegalBargmann transform, described in Section 7 , involves the function $\nu$ evaluated at time $\tau$ and radius $p$.

The resolution of the identity for the coherent states will be obtained by continuously varying the dimensionless parameter $\tau$. When $\tau=0$ the coherent states are simply the position eigenvectors, which have a resolution of the identity because the position operators are self-adjoint. We will show that the function $\nu$ satisfies 
the correct differential equation to make the resolution of the identity remain true as we move to non-zero $\tau$.

Theorem 2 is a special case of Theorem 3 of 17], written out more explicitly and re-stated in terms of coherent states instead of the Segal-Bargmann transform. However we give below a self-contained and elementary proof. The case $d=2$ is also described (with a different proof) in 20]. Since $S^{3}=\mathrm{SU}(2)$, the $d=3$ case belongs to the group case, which is found in 1 . See also Section 4.4 of the second entry in $[8]$ for another proof in the $\mathrm{SU}(2)$ case.

We report here the formulas for the function $\nu(s, R)$, which may be found, for example, in 22, Sect. 5.7] or [26, Eq. (8.73)]. Writing $\nu_{d}(s, R)$ to make explicit the dependence on the dimension we have

$$
\begin{aligned}
& \nu_{1}(s, R)=(2 \pi s)^{-1 / 2} e^{-R^{2} / 2 s} \\
& \nu_{2}(s, R)=(2 \pi s)^{-1} e^{-s / 8} \frac{1}{\sqrt{\pi s}} \int_{R}^{\infty} \frac{\rho e^{-\rho^{2} / 2 s}}{(\cosh \rho-\cosh R)^{1 / 2}} d \rho \\
& \nu_{3}(s, R)=(2 \pi s)^{-3 / 2} e^{-s / 2} \frac{R}{\sinh R} e^{-R^{2} / 2 s} .
\end{aligned}
$$

and the recursion relation

$$
\nu_{d+2}(s, R)=-\frac{e^{-d s / 2}}{2 \pi \sinh R} \frac{\partial}{\partial R} \nu_{d}(s, R) .
$$

Estimates on the behavior as $R \rightarrow \infty$ of $\nu$ may be found in [27, Sect 5.7] and in 28. Note the similarities between the formulas for $\nu$ and the formulas for the heat kernel $\rho_{\tau}$ on the sphere.

Some care must be taken in the interpretation of the integral (44). Even in the $\mathbb{R}^{d}$ case this integral is not absolutely convergent in the operator norm sense. Rather the appropriate sense of convergence is the weak sense. This means that for all vectors $\phi_{1}, \phi_{2}$ in the Hilbert space we have

$$
\left\langle\phi_{1} \mid \phi_{2}\right\rangle=\int_{\mathbf{x} \in S^{d}} \int_{\mathbf{p} \cdot \mathbf{x}=0}\left\langle\phi_{1} \mid \psi_{\mathbf{x}, \mathbf{p}}\right\rangle\left\langle\psi_{\mathbf{x}, \mathbf{p}} \mid \phi_{2}\right\rangle \nu(2 \tau, 2 p)\left(\frac{\sinh 2 p}{2 p}\right)^{d-1} 2^{d} d \mathbf{p} d \mathbf{x}
$$

where the integral (45) is an absolutely convergent complex-valued integral. This of course is formally equivalent to (44). We will prove Theorem 2 at first without worrying about convergence or other similar technicalities. Then at the end we will explain how such matters can be dealt with.

Proof of Theorem 专. We now write the coherent states as $\left|\psi_{\mathbf{a}}^{\tau}\right\rangle$ to emphasize the dependence on the dimensionless quantity $\tau=\hbar / m \omega r^{2}$. We regard the coherent states $\left|\psi_{\mathbf{a}}^{\tau}\right\rangle$ as living in some fixed ( $\tau$-independent) Hilbert space (for example, $\left.L^{2}\left(S^{d}\right)\right)$ and given heuristically by

$$
\left|\psi_{\mathbf{a}}^{\tau}\right\rangle=e^{-\tau \tilde{J}^{2} / 2}\left|\delta_{\mathbf{a}}\right\rangle, \quad \mathbf{a} \in S_{\mathbb{C}}^{d}
$$

where $\left|\delta_{\mathbf{a}}\right\rangle$ is a position eigenvector. Our strategy is essentially the one proposed by T. Thiemann in a more general setting in [7, Sect. 2.3]. We begin with two lemmas that allow us to carry out this strategy explicitly in this situation. The proofs of these lemmas are given at the end of the proof of Theorem 2 .

Lemma 3. The measure

$$
\left(\frac{\sinh 2 p}{2 p}\right)^{d-1} 2^{d} d \mathbf{p} d \mathbf{x}
$$


is invariant under the action of $\mathrm{SO}(d+1 ; \mathbb{C})$ on $S_{\mathbb{C}}^{d} \cong T^{*}\left(S^{d}\right)$.

Lemma 4. Let $J_{\mathbf{a}}^{2}$ and $J_{\overline{\mathbf{a}}}^{2}$ denote the differential operators on $S_{\mathbb{C}}^{d}$ given by

$$
\begin{aligned}
& J_{\mathbf{a}}^{2}=-\sum_{k<l}\left(a_{l} \frac{\partial}{\partial a_{k}}-a_{k} \frac{\partial}{\partial a_{l}}\right)^{2} \\
& J_{\overline{\mathbf{a}}}^{2}=-\sum_{k<l}\left(\bar{a}_{l} \frac{\partial}{\partial \bar{a}_{k}}-\bar{a}_{k} \frac{\partial}{\partial \bar{a}_{l}}\right)^{2} .
\end{aligned}
$$

Let $\phi$ be a smooth, even, real-valued function on $\mathbb{R}$ and consider the function on $S_{\mathbb{C}}^{d}$ given by

$$
\phi(2 p)
$$

where $p$ is regarded as a function of a by means of $(18)$. Then

$$
\begin{array}{r}
J_{\mathbf{a}}^{2} \phi(2 p)=J_{\overline{\mathbf{a}}}^{2} \phi(2 p) \\
=-\left[\frac{\partial^{2} \phi}{\partial R^{2}}+(d-1) \frac{\cosh R}{\sinh R} \frac{\partial \phi}{\partial R}\right]_{R=2 p} .
\end{array}
$$

Assuming for now the two lemmas, we proceed with the proof of the resolution of the identity. Because the coherent states depend holomorphically on a they satisfy

$$
J_{\overline{\mathbf{a}}}^{2}\left|\psi_{\mathbf{a}}^{\tau}\right\rangle=0
$$

Furthermore, it follows from the definition of the coherent states that

$$
\frac{d}{d \tau}\left|\psi_{\mathbf{a}}^{\tau}\right\rangle=\frac{1}{2} J_{\mathbf{a}}^{2}\left|\psi_{\mathbf{a}}^{\tau}\right\rangle
$$

The proof of this is essentially the standard calculation of the action of $\tilde{J}^{2}$ in the position representation. It then follows that the projection operator $\left|\psi_{\mathbf{a}}^{\tau}\right\rangle\left\langle\psi_{\mathbf{a}}^{\tau}\right|$ satisfies the operator-valued differential equation

$$
\frac{\partial}{\partial \tau}\left|\psi_{\mathbf{a}}^{\tau}\right\rangle\left\langle\psi_{\mathbf{a}}^{\tau}\left|=\frac{1}{2}\left(J_{\mathbf{a}}^{2}+J_{\overline{\mathbf{a}}}^{2}\right)\right| \psi_{\mathbf{a}}^{\tau}\right\rangle\left\langle\psi_{\mathbf{a}}^{\tau}\right| .
$$

Now we let

$$
\beta(p)=2^{d}\left(\frac{\sinh 2 p}{2 p}\right)^{d-1} .
$$

Since by Lemma 3 the measure $\beta(p) d \mathbf{p} d \mathbf{x}$ is invariant under the action of $\mathrm{SO}(d+$ $1 ; \mathbb{C})$ the operators $J_{\mathbf{a}}^{2}$ and $J_{\overline{\mathbf{a}}}^{2}$ are self-adjoint in $L^{2}\left(S_{\mathbb{C}}^{d}, \beta(p) d \mathbf{p} d \mathbf{x}\right)$. Thus differentiating under the integral sign and using the self-adjointness gives

$$
\begin{aligned}
& \frac{d}{d \tau} \int_{\mathbf{x} \in S^{d}} \int_{\mathbf{p} \cdot \mathbf{x}=0}\left|\psi_{\mathbf{a}}^{\tau}\right\rangle\left\langle\psi_{\mathbf{a}}^{\tau}\right| \nu(2 \tau, 2 p) \beta(p) d \mathbf{p} d \mathbf{x} \\
& =\int_{\mathbf{x} \in S^{d}} \int_{\mathbf{x} \cdot \mathbf{p}=0}\left|\psi_{\mathbf{a}}^{\tau}\right\rangle\left\langle\psi_{\mathbf{a}}^{\tau}\right|\left[\frac{\partial}{\partial \tau}+\frac{1}{2}\left(J_{\mathbf{a}}^{2}+J_{\overline{\mathbf{a}}}^{2}\right) \nu(2 \tau, 2 p)\right] \beta(p) d \mathbf{p} d \mathbf{x} .
\end{aligned}
$$

Lemma 4 and the differential equation satisfied by $\nu(s, R)$ tell us that the last integral is zero. Thus the operator on the right in (44) is independent of $\tau$. On the other hand, the initial conditions for $\nu$ imply that as $\tau$ tends to zero the measure $\nu(2 \tau, 2 p) \beta(p) d \mathbf{p} d \mathbf{x}$ "collapses" to the Lebesgue measure $d \mathbf{x}$ on the real sphere, i.e. 
the set where $\mathbf{p}=0$. Furthermore, if we consider coherent states $\left|\psi_{\mathbf{x}, \mathbf{p}}^{\tau}\right\rangle$ with $\mathbf{p}=0$, these become simply $\left|\delta_{\mathbf{x}}\right\rangle$ in the $\tau \downarrow 0$ limit. Thus

$$
\lim _{\tau \downarrow 0} \int_{\mathbf{x} \in S^{d}} \int_{\mathbf{p} \cdot \mathbf{x}=0}\left|\psi_{\mathbf{x}, \mathbf{p}}^{\tau}\right\rangle\left\langle\psi_{\mathbf{x}, \mathbf{p}}^{\tau}\left|\nu(2 \tau, 2 p) \beta(p) d \mathbf{p} d \mathbf{x}=\int_{S^{d}}\right| \delta_{\mathbf{x}}\right\rangle\left\langle\delta_{\mathbf{x}}\right| d \mathbf{x}=I .
$$

Since the value of the first integral is independent of $\tau$ this shows that the integral equals the identity for all $\tau$.

It remains now to prove Lemmas 3 and 1 . We begin with the second lemma.

Proof of Lemma 国. Note that expressions such as $\partial / \partial a_{k}$ do not make sense when applied to a function that is defined only on the complex sphere $S_{\mathbb{C}}^{d}$. So the operator $a_{l} \partial / \partial a_{k}-a_{k} \partial / \partial a_{l}$ (and its complex conjugate) should be interpreted as follows. Given a smooth function $f$ on $S_{\mathbb{C}}^{d}$, extend $f$ smoothly to a neighborhood of $S_{\mathbb{C}}^{d}$, then apply $a_{l} \partial / \partial a_{k}-a_{k} \partial / \partial a_{l}$, and then restrict again to $S_{\mathbb{C}}^{d}$. Since $\left(a_{l} \partial / \partial a_{k}-\right.$ $\left.a_{k} \partial / \partial a_{l}\right) a^{2}=0$ the derivatives are all in directions tangent to $S_{\mathbb{C}}^{d}$. This means that the value of the operator on $S_{\mathbb{C}}^{d}$ is independent of the choice of the extension. It is in this way that $J_{\mathbf{a}}^{2}$ and $J_{\overline{\mathbf{a}}}^{2}$ are to be interpreted as operators on $S_{\mathbb{C}}^{d}$.

Now let $R=2 p$ and let $\alpha=|\mathbf{a}|^{2}=\Sigma\left|a_{k}\right|^{2}$. Then (18) (with $r=m \omega=1$ ) tells us that

$$
\alpha:=|\mathbf{a}|^{2}=\cosh ^{2} p+\sinh ^{2} p=\cosh 2 p .
$$

So $R=2 p=\cosh ^{-1} \alpha$. We now need to apply $J_{\mathbf{a}}^{2}$ to a function of the form $\phi(R)$, which we do by using the chain rule

$$
\frac{\partial \phi}{\partial a_{k}}=\frac{d \phi}{d R} \frac{d R}{d \alpha} \frac{\partial \alpha}{\partial a_{k}}
$$

Calculation shows that (for $k \neq l$ )

$$
\begin{aligned}
\left(a_{l} \frac{\partial}{\partial a_{k}}-a_{k} \frac{\partial}{\partial a_{l}}\right)^{2} \phi(R) & =\frac{\left(a_{k} \bar{a}_{l}-a_{l} \bar{a}_{k}\right)^{2}}{|\mathbf{a}|^{4}-1} \frac{\partial^{2} \phi}{\partial R^{2}} \\
& -\frac{\left(\left|a_{k}\right|^{2}+\left|a_{l}\right|^{2}\right)\left(|\mathbf{a}|^{4}-1\right)+|\mathbf{a}|^{2}\left(a_{k} \bar{a}_{l}-a_{l} \bar{a}_{k}\right)^{2}}{\left(|\mathbf{a}|^{4}-1\right)^{3 / 2}} \frac{\partial \phi}{\partial R}
\end{aligned}
$$

We now note that

$$
\begin{aligned}
\sum_{k<l}\left(\left|a_{k}\right|^{2}+\left|a_{l}\right|^{2}\right) & =\frac{1}{2} \sum_{k, l}\left(1-\delta_{k l}\right)\left(\left|a_{k}\right|^{2}+\left|a_{l}\right|^{2}\right) \\
& =\frac{1}{2}\left[2(d+1)|\mathbf{a}|^{2}-2|\mathbf{a}|^{2}\right]=d|\mathbf{a}|^{2} .
\end{aligned}
$$

We use also the easily verified identity

$$
\begin{aligned}
\sum_{k<l}\left(a_{k} \bar{a}_{l}-a_{l} \bar{a}_{k}\right)^{2} & =-\left(|\mathbf{a}|^{4}-\left|a^{2}\right|^{2}\right) \\
& =-\left(|\mathbf{a}|^{4}-1\right),
\end{aligned}
$$

where the first line is valid everywhere and the second line is valid on the complex sphere $S_{\mathbb{C}}^{d}=\left\{a^{2}=1\right\}$. 
Using (49) and (50) we get, upon summing (48) over $k<l$,

$$
\sum_{k<l}\left(a_{l} \frac{\partial}{\partial a_{k}}-a_{k} \frac{\partial}{\partial a_{l}}\right)^{2} \phi(R)=-\frac{\partial^{2} \phi}{\partial R^{2}}-(d-1) \frac{|\mathbf{a}|^{2}}{\sqrt{|\mathbf{a}|^{4}-1}} \frac{\partial \phi}{\partial R} .
$$

Recalling that $|\mathbf{a}|^{2}=\cosh R$, so that $\sqrt{|\mathbf{a}|^{4}-1}=\sinh R$, we get the claimed formula. This completes the proof of the second lemma (with the same argument for the conjugated case).

Proof of Lemma S. Our proof is indirect and uses Lemma 1 . We regard $S_{\mathbb{C}}^{d}$ as the quotient $\mathrm{SO}(d+1 ; \mathbb{C}) / \mathrm{SO}(d ; \mathbb{C})$. Since both $\mathrm{SO}(d+1 ; \mathbb{C})$ and $\mathrm{SO}(d ; \mathbb{C})$ are unimodular, general principles [29, Thm. 8.36] tell us that there is a smooth $\mathrm{SO}(d+$ $1 ; \mathbb{C})$-invariant measure on $S_{\mathbb{C}}^{d}$ and that it is unique up to a constant. This measure must be in particular $\mathrm{SO}(d+1)$-invariant, which means that it must be of the form $\gamma(p) d \mathbf{p} d \mathbf{x}$, since $d \mathbf{p} d \mathbf{x}$ is also $\mathrm{SO}(d+1)$-invariant. Now the operator $J_{\mathbf{a}}^{2}$ must be self-adjoint with respect to the $\mathrm{SO}(d+1 ; \mathbb{C})$-invariant measure. In particular, $J_{\mathbf{a}}^{2}$ must be self-adjoint when restricted to the space of $\mathrm{SO}(d+1)$-invariant functions, which can all be written in the form $f(\mathbf{a})=\phi(2 p)$, as in Lemma 4 .

Meanwhile, according to Lemma 4, on $\mathrm{SO}(d+1)$-invariant functions $J_{\mathbf{a}}^{2}$ is just the hyperbolic Laplacian, re-scaled by a factor of 2 . This operator is therefore selfadjoint (on $\mathrm{SO}(d+1$ )-invariant functions) with respect to the measure $\beta(p) d \mathbf{p} d \mathbf{x}$, which is just hyperbolic volume measure with the same re-scaling.

We conclude, then, that on $\mathrm{SO}(d+1)$-invariant functions, $J_{\mathbf{a}}^{2}$ is self-adjoint with respect to both the measures $\gamma(p) d \mathbf{p} d \mathbf{x}$ and $\beta(p) d \mathbf{p} d \mathbf{x}$. From this it follows that

$$
\left[\frac{\partial^{2} g}{\partial R^{2}}+(d-1) \frac{\cosh R}{\sinh R} \frac{\partial g}{\partial R}\right]_{R=2 p}=0
$$

where $g(p)=\gamma(p) / \beta(p)$. But since both $\gamma$ and $\beta$ are smooth, $\mathrm{SO}(d+1)$-invariant functions on $S_{\mathbb{C}}^{d}$ we must have $\partial g /\left.\partial R\right|_{R=0}=0$. Solving (51) gives $\partial g / \partial R=$ $c \exp \left[-(d-1) \int \operatorname{coth} S d S\right]$, so we have

$$
\left.\frac{\partial g}{\partial R}\right|_{R=0}=c \lim _{\varepsilon \rightarrow 0} \exp \left[(d-1) \int_{\varepsilon}^{1} \operatorname{coth} S d S\right]=0,
$$

which can occur only if $c=0$, i.e. if $g$ is constant. Thus $\gamma$ is a constant multiple of $\beta$, which is what we want to show.

We conclude this section with a few remarks about technicalities in the proof of the resolution of the identity. We have already said that the integral in Theorem 2 is to be interpreted in the weak sense, as in (45). We first establish (45) in the case where $\left|\phi_{1}\right\rangle$ and $\left|\phi_{2}\right\rangle$ are finite linear combinations of spherical harmonics. In that case it can be shown that the integrand $\left\langle\phi_{1} \mid \psi_{\mathbf{x}, \mathbf{p}}^{\tau}\right\rangle\left\langle\psi_{\mathbf{x}, \mathbf{p}}^{\tau} \mid \phi_{2}\right\rangle$ grows only exponentially with $\mathbf{p}$. Since $\nu$ has faster-than-exponential decay (namely, Gaussian) the integral (45) is convergent. In this case there is not much difficulty in justifying the formal manipulations we have made, such as interchanging derivatives with the integral and integrating by parts. Then once (45) is established for such "nice" vectors, a simple passage to the limit will establish it for all $\left|\phi_{1}\right\rangle$ and $\left|\phi_{2}\right\rangle$ in the Hilbert space. See [1] or [17] for more details on these technicalities. 


\section{The Segal-Bargmann RePresentation}

As shown in Section 9, any two irreducible unitary representations of $\tilde{E}(d+1)$ satisfying (26) and (34) are equivalent. The simplest concrete realization of such representations is the position representation, in which the Hilbert space is $L^{2}\left(S^{d}\right)$, the position operators act by multiplication, and the angular momentum operators act as differential operators given by

$$
J_{k l}=-i \hbar\left(x_{l} \frac{\partial}{\partial x_{k}}-x_{k} \frac{\partial}{\partial x_{l}}\right) .
$$

The resolution of the identity for the coherent states can be used to give another realization, the (generalized) Segal-Bargmann representation. In the SegalBargmann representation the Hilbert space is the space of holomorphic functions on $S_{\mathbb{C}}^{d}$ that are square-integrable with respect to the density occurring in the resolution of the identity. In this representation the action of the position operators $X_{k}$ is somewhat complicated, but the action of the creation operators (the adjoints of the annihilation operators) becomes simply multiplication by $a_{k}$. The resolution of the identity can be re-interpreted as the unitary intertwining map between these two representations, that is, the generalized Segal-Bargmann transform.

Specifically, given any function $f$ in $L^{2}\left(S^{d}\right)$ we define the Segal-Bargmann transform $C f$ of $f$ by

$$
C f(\mathbf{a})=\left\langle\psi_{\overline{\mathbf{a}}} \mid f\right\rangle
$$

Then for any $f, C f(\mathbf{a})$ is a holomorphic function of $\mathbf{a} \in S_{\mathbb{C}}^{d}$. Note that in the interests of consistency with [1, 17] we have put a complex conjugate on the a in the (53), so that the dependence of $C f$ on $\mathbf{a} \in S_{\mathbb{C}}^{d}$ is holomorphic rather than anti-holomorphic. The Segal-Bargmann transform can be computed as

$$
C f(\mathbf{a})=\int_{S^{d}} \rho_{\tau}(\mathbf{a}, \mathbf{x}) f(\mathbf{x}) d \mathbf{x}, \quad \mathbf{a} \in S_{\mathbb{C}}^{d} .
$$

Here $\rho_{\tau}(\mathbf{a}, \mathbf{x})$ is the heat kernel on $S^{d}$, with the a variable extended by analytic continuation from $S^{d}$ to $S_{\mathbb{C}}^{d}$.

Theorem 5 (Segal-Bargmann transform). The map $C$ defined by (53) or (54) is a unitary map of $L^{2}\left(S^{d}, d \mathbf{x}\right)$ onto $\mathcal{H} L^{2}\left(S_{\mathbb{C}}^{d}, \nu\right)$, where $\mathcal{H} L^{2}\left(S_{\mathbb{C}}^{d}, \nu\right)$ denotes the space of holomorphic functions $F$ on $S_{\mathbb{C}}^{d}$ for which

$$
\int_{\mathbf{x} \in S^{d}} \int_{\mathbf{p} \cdot \mathbf{x}=0}|F(\mathbf{a}(\mathbf{x}, \mathbf{p}))|^{2} \nu(2 \tau, 2 p)\left(\frac{\sinh 2 p}{2 p}\right)^{d-1} 2^{d} d \mathbf{p} d \mathbf{x}<\infty .
$$

The isometricity of the map $C$ as a map from $L^{2}\left(S^{d}\right)$ into $L^{2}\left(S_{\mathbb{C}}^{d}, \nu\right)$ is equivalent to the resolution of the identity. (Compare (45).) That $C$ maps into the holomorphic subspace of $L^{2}\left(S_{\mathbb{C}}^{d}, \nu\right)$ follows from the holomorphic dependence of the coherent states on a. It remains only to show that the image of $C$ is all of $\mathcal{H} L^{2}\left(S_{\mathbb{C}}^{d}, \nu\right)$. The proof of this is a fairly straightforward density argument using spherical harmonics, which we omit. (See Section 4 of [17] and Section 8 of [1].)

In the Segal-Bargmann space $\mathcal{H} L^{2}\left(S_{\mathbb{C}}^{d}, \nu\right)$ the angular momentum operators act by the holomorphic analog of (52), namely,

$$
J_{k l}=-i \hbar\left(a_{l} \frac{\partial}{\partial a_{k}}-a_{k} \frac{\partial}{\partial a_{l}}\right) .
$$


Meanwhile, the creation operators, defined as the adjoints of the annihilation operators, are given by

$$
A_{k}^{\dagger} F(\mathbf{a})=a_{k} F(\mathbf{a})
$$

The annihilation operators can be described as Toeplitz operators. This means that

$$
A_{k} F=P\left(\bar{a}_{k} F\right)
$$

where $P$ is the orthogonal projection from the full $L^{2}$-space $L^{2}\left(S_{\mathbb{C}}^{d}, \nu\right)$ onto the holomorphic subspace. (See [33].) In the Segal-Bargmann representation the action of the position operators is more complicated.

Another important feature of the Segal-Bargmann representation is the reproducing kernel identity. Recall from Section 5 that the reproducing kernel $R_{\tau}(\mathbf{a}, \mathbf{b})=$ $\left\langle\psi_{\mathbf{b}} \mid \psi_{\mathbf{a}}\right\rangle$ is holomorphic in $\mathbf{a}$ and anti-holomorphic in $\mathbf{b}$. We then have the following result, which follows easily from general principles. (See, for example, Section 9 of [1.)

Proposition 6 (Reproducing kernel identity). For any $F \in \mathcal{H} L^{2}\left(S_{\mathbb{C}}^{d}, \nu\right)$ we have

$$
F(\mathbf{a})=\int_{\mathbf{x} \in S^{d}} \int_{\mathbf{p} \cdot \mathbf{x}=0} R_{\tau}(\mathbf{a}, \mathbf{b}) F(\mathbf{b}(\mathbf{x}, \mathbf{p})) \nu(2 \tau, 2 p)\left(\frac{\sinh 2 p}{2 p}\right)^{d-1} 2^{d} d \mathbf{p} d \mathbf{x} .
$$

Here $R_{\tau}(\mathbf{a}, \mathbf{b})=\rho_{2 \tau}(\mathbf{a}, \overline{\mathbf{b}})$ is the reproducing kernel, and the integral is absolutely convergent.

The Segal-Bargmann representation can be thought of as defining a phase space wave function for a quantum particle on the sphere, which is related to the position wave function by the Segal-Bargmann transform. This phase space wave function can then be turned into a phase space probability density in the usual way: if $f$ is a unit vector in $L^{2}\left(S^{d}\right)$ then the associated probability density is

$$
|C f(\mathbf{a}(\mathbf{x}, \mathbf{p}))|^{2} \nu(2 \tau, 2 p)\left(\frac{\sinh 2 p}{2 p}\right)^{d-1} 2^{d} .
$$

This is a manifestly non-negative function on the phase space that integrates to one. In the $\mathbb{R}^{d}$ case [33], the expression corresponds to the Husimi function of $f$.

If one takes the probability density (55) and integrates out the momentum variables one will not get the standard position probability density $|f(\mathbf{x})|^{2}$ (even in the $\mathbb{R}^{d}$ case). That is, with this definition, the position probability density cannot be obtained from the phase space probability density by integrating out the momentum variables. On the other hand, there is a nice inversion formula for the generalized Segal-Bargmann transform that can be stated roughly as follows: the position wave function can be obtained from the phase space wave function by integrating out the momentum variables.

Theorem 7 (Inversion Formula). Given any function $f$ in $L^{2}\left(S^{d}\right)$, let $F=C f$ be the Segal-Bargmann transform of $f$. Then $f$ may be recovered from $F$ by the formula

$$
f(\mathbf{x})=\int_{\mathbf{p} \cdot \mathbf{x}=0} F(\mathbf{a}(\mathbf{x}, \mathbf{p})) \nu(\tau, p)\left(\frac{\sinh p}{p}\right)^{d-1} d \mathbf{p} .
$$

This result is a special case of [17]; the group analog of this inversion formula was given in [3]. An analog of this formula holds also in the $\mathbb{R}^{d}$ case $[$, Sect. 4], but 
does not seem to be well known. Note that whereas the resolution of the identity involves $\nu(2 \tau, 2 p)$, the inversion formula involves $\nu(\tau, p)$.

This statement of the inversion formula is a bit imprecise, because we have glossed over the question of the convergence of the integral in (56). The integral cannot always be convergent, since a general function $f$ in $L^{2}\left(S^{d}\right)$ can have singularities. As shown in Theorems 1 and 2 of 17, we have the following two precise statements. First, if $f$ is sufficiently smooth, then the integral in $(56)$ is absolutely convergent for all $\mathbf{x}$ and is equal to $f(\mathbf{x})$. Second, for any $f \in L^{2}\left(S^{d}\right)$ we have

$$
f(\mathbf{x})=\lim _{R \rightarrow \infty} \int_{\substack{\mathbf{p} \cdot \mathbf{x}=0 \\ p \leq R}} F(\mathbf{a}(\mathbf{x}, \mathbf{p})) \nu(\tau, p)\left(\frac{\sinh p}{p}\right)^{d-1} d \mathbf{p}
$$

where the limit is in the topology of $L^{2}\left(S^{d}\right)$.

We will describe the proof of Theorem 7 in greater detail in the setting of general compact symmetric spaces. Here we give only the barest outline. The CauchyRiemann equations on $S_{\mathbb{C}}^{d}$ imply that, when applied to holomorphic functions, the hyperbolic Laplacian in the momentum variables is the negative of the spherical Laplacian in the position variables, just as for holomorphic functions on $\mathbb{C}$ we have $\partial^{2} F / \partial y^{2}=-\partial^{2} F / \partial x^{2}$. For this result to hold, we must omit the re-scaling of the momentum variables that is present in the resolution of the identity; hence the inversion formula involves $\nu(\tau, p)$ rather than $\nu(2 \tau, 2 p)$. The integration in (56) against the hyperbolic heat kernel is computing the forward heat equation in the momentum variables, which for holomorphic functions is then the same as the backward heat equation in the position variables. Since the Segal-Bargmann transform may be computed in terms of the forward heat equation in the position variables, (56) is inverting the Segal-Bargmann transform. Although this is the basic idea of the proof, the convergence questions are more subtle.

Note that there are, besides (56), many other inversion formulas for the SegalBargmann transform. The "overcompleteness" of the coherent states means that there is a lot of redundant information in the Segal-Bargmann transform, and therefore many different ways that one can recover $f$ from $C f$. To look at it another way, it is possible to have many different integrals that all give the same value when applied to holomorphic functions, as in the Cauchy integral formula. Of particular importance is the inversion formula

$$
f\left(\mathbf{x}^{\prime}\right)=\int_{S_{\mathbb{C}}^{d}} \overline{\rho_{\tau}\left(\mathbf{a}, \mathbf{x}^{\prime}\right)} F(\mathbf{a}(\mathbf{x}, \mathbf{p})) \nu(2 \tau, 2 p)\left(\frac{\sinh 2 p}{2 p}\right)^{d-1} 2^{d} d \mathbf{p} d \mathbf{x},
$$

where $\rho_{\tau}$ is the analytical continuation of the heat kernel for $S^{d}$. This formula is obtained by noting that $C$ is isometric, and therefore its inverse is its adjoint. One can apply the above integral to any function $F$ in $L^{2}\left(S_{\mathbb{C}}^{d}, \nu\right)$ (not necessarily holomorphic), in which case we have $f=C^{-1} P F$, where $P F$ is the orthogonal projection of $F$ onto the holomorphic subspace of $L^{2}\left(S_{\mathbb{C}}^{d}, \nu\right)$. See [1, Sect. 9] and 20. Eq. (6.13)].

\section{THE $\mathbb{R}^{d}$ CASE}

We verify in this section that the methods in this paper, when applied to the $\mathbb{R}^{d}$ case, do indeed reproduce the canonical coherent states. Our "complexifier" is $1 / \omega$ times the kinetic energy function, namely, $p^{2} / 2 m \omega$. (In the $\mathbb{R}^{d}$ case the kinetic 
energy cannot be expressed in terms of the angular momentum.) Then we define

$$
\begin{aligned}
a_{k} & =e^{i\{\cdot, \text { complexifier }\}} x_{k} \\
& =\sum_{n=0}^{\infty}\left(\frac{i}{2 m \omega}\right)^{n} \frac{1}{n !} \underbrace{\left\{\cdots\left\{\left\{x_{k}, p^{2}\right\}, p^{2}\right\}, \cdots, p^{2}\right\}}_{n} .
\end{aligned}
$$

Since $\left\{x_{k}, p^{2}\right\}=2 p_{k}$ and $\left\{\left\{x_{k}, p^{2}\right\}, p^{2}\right\}=0$ we obtain

$$
a_{k}=x_{k}+i \frac{p_{k}}{m \omega} \text {. }
$$

This is, up to an overall constant, the standard complex coordinate on phase space. More generally one can apply the same method to any function of the $x_{k}$ 's, and one will obtain the corresponding function of $a_{k}$. For example, it is easily verified by induction that

$$
e^{i\{\cdot, \text { complexifier }\}}\left(x_{k}^{n}\right)=\left(x_{k}+i \frac{p_{k}}{m \omega}\right)^{n}
$$

for all positive integers $n$.

Similarly on the quantum side if we define the complexifier to be $P^{2} / 2 m \omega$ and

$$
\begin{aligned}
A_{k} & =e^{i[\cdot, \text { complexifier }] / i \hbar} X_{k} \\
& =\sum_{n=0}^{\infty} \frac{1}{(2 m \omega \hbar)^{n}} \frac{1}{n !}\left[\cdots\left[\left[X_{k}, P^{2}\right], P^{2}\right] \cdots, P^{2}\right]
\end{aligned}
$$

we get simply

$$
A_{k}=X_{k}+i \frac{P_{k}}{m \omega} .
$$

This is, up to an overall constant, the usual annihilation operator. Applying the same procedure to any function of the $X_{k}$ 's will give the corresponding function of the $A_{k}$ 's.

Following the same normalization procedure as in the sphere case we obtain coherent states given by

$$
\left|\psi_{\mathbf{a}}\right\rangle=e^{-P^{2} / 2 m \omega \hbar}\left|\delta_{\mathbf{a}}\right\rangle
$$

at first for $\mathbf{a} \in \mathbb{R}^{d}$ and then by analytic continuation for any $\mathbf{a} \in \mathbb{C}^{d}$. In the $\mathbb{R}^{d}$ case we have the formula

$$
\left|\psi_{\mathbf{a}}\right\rangle=e^{i \mathbf{a} \cdot \mathbf{P} / \hbar}\left|\psi_{0}\right\rangle
$$

This normalization coincides with what Hecht [30] calls Type I coherent states. In the position representation we have

$$
\left\langle\delta_{\mathbf{x}} \mid \psi_{\mathbf{a}}\right\rangle=(2 \pi \hbar / m \omega)^{-d / 2} \exp \left[-\frac{(\mathbf{x}-\mathbf{a})^{2}}{2 \hbar / m \omega}\right] .
$$

With this normalization of the coherent states the resolution of the identity takes the form

$$
I=\int_{\mathbb{C}^{d}}\left|\psi_{\mathbf{a}}\right\rangle\left\langle\psi_{\mathbf{a}}\right| \gamma(\mathbf{a}) d \mathbf{a}
$$

where $d \mathbf{a}$ is $2 d$-dimensional Lebesgue measure and where $\gamma$ is the density

$$
\gamma(\mathbf{a})=\left(\frac{\pi \hbar}{m \omega}\right)^{-d / 2} \exp \left[-\frac{(\operatorname{Im} \mathbf{a})^{2}}{\hbar / m \omega}\right] \text {. }
$$


The associated Segal-Bargmann space is the space of holomorphic functions on $\mathbb{C}^{d}$ that are square-integrable with respect to the density $\gamma$. This normalization of the Segal-Bargmann space is different from that of Segal [31] and Bargmann [32], because of the different normalization of the coherent states. See [33, Sect. 6] for comparisons with the conventions of Segal and of Bargmann.

To compare this to what we have in the sphere case, let $\sigma=\hbar / m \omega$ and consider the Euclidean heat kernel in the imaginary directions, given by

$$
\nu(\sigma, \mathbf{a})=(2 \pi \sigma)^{-d / 2} \exp \left[-\frac{(\operatorname{Im} \mathbf{a})^{2}}{2 \sigma}\right] .
$$

Then $\gamma(\mathbf{a})=2^{d} \nu(2 \sigma, 2 \mathbf{a})$, similar to what we have in the sphere case. Note that in the Euclidean case $\nu(2 \sigma, 2 \mathbf{a})$ is the same, up to an overall constant, as $\nu(\sigma / 2, \mathbf{a})$. Thus it is hard to see the "correct" scaling of the space and time variables from the Euclidean case.

An inversion formula similar to Theorem 7 holds in the $\mathbb{R}^{d}$ case; see 4 , Sect. 4].

\section{Representation theORY OF the EuClidean Group}

We consider representations by self-adjoint operators of the commutation relations (25) for the Lie algebra e $(d+1)$. We further assume that these operators are the Lie algebra representation associated to a representation of the corresponding connected, simply connected Lie group $\tilde{\mathrm{E}}(d+1)$. It is known that all the irreducible unitary representations of $\tilde{\mathrm{E}}(d+1)$ can be realized in spaces of sections of smooth vector bundles with the Lie algebra acting by smooth differential operators. The action of the Lie algebra then extends to an action on distributional sections, including the generalized eigenvectors of the position operators. With this discussion in mind we will make free use of position eigenvectors in what follows.

We apply the Wigner-Mackey method and consider an orbit of Spin $(d+1)$ in $\mathbb{R}^{d+1}$, namely, a sphere of radius $r$. We consider only the case $r>0$, in which case the little group is $\operatorname{Spin}(d)$. Fixing a value for $r$ amounts to assuming that the operators $X_{k}$ satisfy $\sum X_{k}^{2}=r^{2}$.

The purpose of this section is to show that the little group acts trivially if and if the following relation holds for all $k$ and $l$ :

$$
X^{2} J_{k l}=J_{k m} X_{m} X_{l}-J_{l m} X_{m} X_{k}
$$

(sum convention). This is equivalent to the relation

$$
J_{k l}=P_{k} X_{l}-P_{l} X_{k}
$$

where by definition $P_{k}=r^{-2} J_{k l} X_{l}$.

Note that (57) is the quantum counterpart of the constraint to the sphere (9) and therefore representations of $\tilde{\mathrm{E}}(d+1)$ satisfying it are closest to the classical motion on a sphere. Nevertheless, other representations are of interest, and describe a quantum particle on a sphere with internal degrees of freedom. We will consider the general case in a future work.

Suppose now that (57) holds. We wish to show that this implies that the representation of the little group is trivial. So we consider the space of generalized eigenvectors for the operators $X_{k}$ satisfying

$$
\begin{aligned}
X_{k}|\psi\rangle & =0, \quad k=1, \cdots, d \\
X_{d+1}|\psi\rangle & =r .
\end{aligned}
$$


This is the space on which the little group acts, where the Lie algebra of the little group is given by the operators $J_{k l}$ with $1 \leq k, l \leq d$. But now if (57) holds then for $k, l \leq d$ we have

$$
r^{2} J_{k l}|\psi\rangle=0
$$

since in that case $X_{k}|\psi\rangle=X_{l}|\psi\rangle=0$. This shows (for $r>0$ ) that if (57) holds then the little group acts trivially.

Consider now the quantity

$$
W_{k l}:=X^{2} J_{k l}-J_{k m} X_{m} X_{l}+J_{l m} X_{m} X_{k},
$$

which satisfies $W_{l k}=-W_{k l}$. The condition (57) is equivalent to $W_{k l}=0$. Consider also the quantity

$$
C:=\sum_{k<l} W_{k l}^{2}
$$

As we will show below, $C$ is a Casimir, that is, an element of the universal enveloping algebra of e $(d+1)$. This implies that $C$ acts as $c I$ in each irreducible representation. (The value of the constant $c$ is $r^{4}$ times the value of the quadratic Casimir for the little group in each generalized eigenspace for the position operators.)

Let us now assume that the little group acts trivially and determine the value of $c$ in this case. We may compute $c$ by applying $C$ to a position eigenvector as in (59). That the little group acts trivially means that $J_{k l}|\psi\rangle=0$ for $k<l<d+1$. Since also $X_{k}|\psi\rangle=X_{l}|\psi\rangle=0$ for $k<l<d+1$ we get

$$
C|\psi\rangle=c|\psi\rangle=\sum_{k}\left(X^{2} J_{k, d+1}-J_{k m} X_{m} X_{d+1}+J_{d+1, m} X_{m} X_{k}\right)^{2}|\psi\rangle .
$$

But since $X_{m}|\psi\rangle=0$ unless and $m=d+1$ (and since $J_{d+1, d+1}=0$ ) we get that

$$
\begin{aligned}
\left(X^{2} J_{k, d+1}-J_{k m} X_{m} X_{d+1}+J_{d+1, m} X_{m} X_{k}\right)|\psi\rangle & =\left(X^{2} J_{k, d+1}-J_{k, d+1} X_{d+1}^{2}+0\right)|\psi\rangle \\
& =\left(r^{2} J_{k, d+1}-r^{2} J_{k, d+1}\right)|\psi\rangle \\
& =0 .
\end{aligned}
$$

This means that if the representation of the little group is trivial then the constant $c$ must be zero, which means the element $C$ must be zero in that representation. A calculation shows that for each $k<l, W_{k l}$ is self-adjoint. Thus $C$ is a sum of squares of self-adjoint operators, and the only way the sum can be zero is if each term is zero, that is, if (57) holds. So if the little group acts trivially, (57) must hold, which is what we want to prove.

In the case $d=2$ (considered in [18) it is possible to verify that

$$
C=X^{2}(L \cdot X)^{2},
$$

where $L$ is the angular momentum vector, related to our angular momentum matrix by $L=\left(J_{32}, J_{13}, J_{12}\right)$. One can easily check that at least this relation holds in each irreducible representation (which is all that is really relevant) as follows. Both sides are Casimirs and so it suffices to check (62) on the generalized eigenspace in (59). But for $|\psi\rangle$ in this space we calculate that

$$
C|\psi\rangle=X^{2}(L \cdot X)^{2}|\psi\rangle=X^{4} J_{12}^{2}|\psi\rangle,
$$

and indeed (62) holds. From (62) we see that taking $C=0$ is equivalent in the $d=2$ case to taking $L \cdot X=0$ as in [18]. 
It remains only to show that the element $C$ in (61) is a Casimir. To do this we first compute the commutation relations of $W_{k l}$ with the $J$ 's and the $X$ 's. These come out to be

$$
\begin{aligned}
& \frac{1}{i \hbar}\left[X_{k}, W_{l m}\right]=0 \\
& \frac{1}{i \hbar}\left[J_{k l}, W_{m n}\right]=\delta_{k n} W_{l m}+\delta_{l m} W_{k n}-\delta_{k m} W_{l n}-\delta_{l n} W_{k m} .
\end{aligned}
$$

Equation (64) is what we expect for a matrix operator-compare this to the formula for $\left[J_{k l}, J_{m n}\right]$. Equation (63) implies immediately that $C$ commutes with each $X_{k}$, and Equation (64) implies, after a short calculation, that $C$ commutes with each $J_{k l}$.

\section{CONCLUding REMARKS}

We end this paper by discussing how the coherent states described here compare to other coherent states that have been proposed for systems whose configuration space is a sphere (or homogeneous space). As we have explained in detail above, the coherent states introduced in 18 are equivalent to those in [1], 17], but were discovered independently and from a different point of view.

Meanwhile, there are several other generalized Segal-Bargmann transforms for spheres that have been considered. These are similar but not identical to each other and were introduced by Bargmann and Todorov [34, Rawnsley [35], Ii [36], Wada [37, Thomas and Wassell [38, and Villegas [39]. In most cases the transform is unitary, and this unitarity can be re-formulated as a resolution of the identity for the associated coherent states. These constructions all have in common that the coherent states are labeled by points in the cotangent bundle minus the zero section (i.e. with the points of zero momentum removed). In these papers the cotangent bundle minus the zero section is identified with the null quadric $\left\{a \in \mathbb{C}^{d+1} \mid a^{2}=0\right\}$. This is to be contrasted with the present paper, in which the full cotangent bundle of the sphere is identified with the quadric $\left\{a^{2}=r^{2}\right\}$ with $r>0$. Thus these constructions are inequivalent to the one considered in this paper. Furthermore these constructions do not generalize to higher-rank symmetric spaces [40].

Besides these, there have been to our knowledge two other proposed constructions of coherent states on spheres (and other homogeneous spaces). These constructions, inequivalent to [1, 17] and to each other, are those of S. De Bièvre [41] and of C. Isham and J. Klauder [42. Both [41] and 42] are based on extensions of the Perelomov approach, in that their coherent states are all obtained from one fixed vector $\psi_{0}$ by the action of the Euclidean group. As explained in those papers, the ordinary Perelomov approach is not applicable in this case, because the irreducible representations of the Euclidean group are not square-integrable. Nonsquare-integrability means that the usual Perelomov-type integral, which should be a multiple of the identity operator, is in this case divergent.

De Bièvre's approach to this problem is to apply to the fiducial vector $\psi_{0}$ only a part of the Euclidean group. We describe just the simplest case of 41]. (This special case was worked out independently in a more elementary way by Torresani 43.) Specifically, if we work in $L^{2}\left(S^{d}\right)$ then start with a basic coherent state $\psi_{0}$ such that a) $\psi_{0}$ is invariant under rotations about the north pole $\mathbf{n}$ and b) $\psi_{0}$ is supported in the northern half-sphere with a certain rate of decay at the equator. One may think of $\psi_{0}$ being concentrated near the north pole and approximating a 
state whose position is at the north pole and whose momentum is zero. The other coherent states are then of the form

$$
\exp (i \mathbf{k} \cdot \mathbf{x}) \psi_{0}\left(R^{-1} \mathbf{x}\right)
$$

where we consider only pairs $(\mathbf{k}, R)$ satisfying $\mathbf{k} \cdot R \mathbf{n}=0$. This last restriction is crucial. Since $\psi_{0}$ is invariant under rotations about the north pole, the coherent states are determined by the values of $\mathbf{k}$ and $R \mathbf{n}$ and are thus labeled by points in the cotangent bundle of $S^{d}$. The resolution of the identity for these coherent states follows from the general procedure in 441 but can also be proved in this case by an elementary application of the Plancherel formula. The condition that $\psi_{0}$ be supported in the northern half-sphere is crucial to the proof.

It is clear that the coherent states considered in this paper are quite different from those in 41]. First, De Bièvre's coherent states do not depend holomorphically on the parameters. Second, each coherent state must be supported in a half-sphere, hence cannot be real-analytic in the space variable. Third, there does not seem to be any preferred choice for $\psi_{0}$ in 41], whereas for the coherent states considered here the only choice one has to make is the value of the parameter $\omega$.

Meanwhile, Isham and Klauder use a different method of working around the non-square-integrability of the irreducible representations of $\mathrm{E}(d+1)$. They use reducible representations, corresponding to integration over some small range $[r, r+\varepsilon]$ of radii. This allows for a family of coherent states invariant under the full Euclidean group and allows a more general basic coherent state $\psi_{0}$, without any support conditions. On the other hand it seems natural to get back to an irreducible representation by letting $\varepsilon$ tend to zero, so that the particle is constrained to a sphere with one fixed radius. Unfortunately, although the representation itself does behave well under this limit (becoming irreducible) the coherent states themselves do not have a limit as $\varepsilon$ tends to zero. (See the remarks at the bottom of the first column on p.609 in 42.) This seems to be a drawback of this approach.

Finally, we mention that in the group case, the coherent states described in this paper can be obtained by means of geometric quantization, as shown in 14 . This means that in the group case the coherent states are of "Rawnsley type" 15$]$. However, this result does not carry over to the case of general compact symmetric spaces. In particular the results of [14] apply only to those spheres that are also groups, namely, $S^{1}=\mathrm{U}(1)$ and $S^{3}=\mathrm{SU}(2)$.

\section{Acknowledgments}

The second author was supported in part by NSF Grant DMS-9970882.

\section{REFERENCES}

[1] B. Hall, J. Funct. Anal. 122, 103 (1994).

[2] B. Hall, Comm. Math. Phys. 184, 233 (1997).

[3] B. Hall, J. Funct. Anal. 143, 98 (1997).

[4] B. Hall, in Perspectives on quantization, edited by L. Coburn and M. Rieffel, (Amer. Math. Soc., Providence, RI, 1998), p. 47.

[5] B. Hall, Bull. (N.S.) Amer. Math. Soc. 38, 43 (2001).

[6] A. Ashtekar, J. Lewandowski, D. Marolf, J. Mourão, and T. Thiemann, J. Funct. Anal. 135, 519 (1996); R. Loll, Nucl. Phys. B 444, 619 (1995).

[7] T. Thiemann, Classical Quantum Gravity 13, 1383 (1996). 
[8] T. Thiemann, Class. Quantum Grav. 18, 2025 (2001); T. Thiemann and O. Winkler, Class. Quantum Grav. 18, 2561 (2001); H. Sahlmann, T. Thiemann and O. Winkler, Nuclear Physics B 606, 401 (2001); T. Thiemann and O. Winkler, Class. Quantum Grav. 18, 4629 (2001)

[9] T. Thiemann, Class. Quantum Grav. 15, 839 (1998); Class. Quantum Grav. 15, 875 (1998); Class. Quantum Grav. 15, 1207 (1998); Class. Quantum Grav. 15, 1249 (1998); Class. Quantum Grav. 15, 1281 (1998); Class. Quantum Grav. 15, 1463 (1998).

[10] K. Wren, Nuclear Phys. B 521, 471 (1998). See also Section IV.3.7 in N. Landsman, Mathematical topics between classical and quantum mechanics, (Springer-Verlag, New York/Berlin, 1998).

[11] N. Landsman, J. Geom. Phys. 15, 285 (1995).

[12] B. Driver and B. Hall, Comm. Math. Phys. 201, 249 (1999); B. Hall, Rev. Math. Phys. 13, 1281 (2001).

[13] S. Albeverio, B. Hall, and A. Sengupta, Infinite Dimensional Anal. Quantum Prob. 2, 27 (1999).

[14] B. Hall, Comm. Math. Phys., to appear, quant-ph/0012105.

[15] J. Rawnsley, Quart. J. Math. Oxford Ser. (2) 28, 403 (1977); J. Rawnsley, M. Cahen, and S. Gutt, J. Geom. Phys. 7, 45 (1990).

[16] A. Perelomov, Generalized coherent states and their applications, (Springer-Verlag, New York/Berlin, 1986).

[17] M. Stenzel, J. Funct. Anal. 165, 44 (1999).

[18] K. Kowalski and J. Rembieliński, J. Phys. A 33, 6035 (2000).

[19] Kowalski, K., Rembieliński, J., and Papaloucas, L., J. Phys. A 29, 4149 (1996).

[20] K. Kowalski and J. Rembieliński, J. Math. Phys. 42, 4138 (2001).

[21] L. Lempert and R. Szőke, Math. Ann. 290, 689 (1991).

[22] V. Guillemin and M. Stenzel, J. Differential Geom. 34, 561 (1991).

[23] A. Morimoto and T. Nagano, J. Math. Soc. Japan 15, 289 (1963).

[24] R. Szöke, Math. Ann. 291, 409 (1991).

[25] M. Taylor, Noncommutative harmonic analysis, (American Math. Soc., Providence, RI, 1986).

[26] R. Camporesi, Phys. Rep. 196, 1 (1990).

[27] E. B. Davies, Heat kernels and spectral theory (Cambridge University Press, Cambridge, 1990).

[28] E. B. Davies and N. Mandouvalos, Proc. London Math. Soc. (3) 57, 182 (1988).

[29] A. Knapp, Lie groups: beyond an introduction, (Birkhäuser, Boston/Berlin, 1996).

[30] K. Hecht, The vector coherent state method and its application to problems of higher symmetries, (Springer-Verlag, New York/Berlin, 1987).

[31] I. Segal, in Proceedings of the Summer Seminar, Boulder, Colorado, 1960, Vol. II, edited by M. Kac, (American Math. Soc., Providence, RI, 1963); I. Segal, Illinois J. Math. 6, 500 (1962); I. Segal, in Topics in functional analysis: Essays dedicated to M.G. Krein on the occasion of his 70th birthday, edited by I. Gohberg and M. Kac, (Academic Press, San Diego, 1978), p, 321.

[32] V. Bargmann, Comm. Pure Appl. Math. 14, 187 (1961).

[33] B. Hall, in First Summer School in Analysis and Mathematical Physics, edited by S. Pérez Esteva and C. Villegas-Blas, (Amer. Math. Soc., Providence, RI, 2000), p. 1.

[34] V. Bargmann and I. Todorov, J. Math. Phys. 18, 1141 (1977).

[35] J. Rawnsley, Trans. Amer. Math. Soc. 250, 167 (1979).

[36] K. Ii, Tohoku Math. J. (2) 38, 57 (1986).

[37] R. Wada, Tohoku Math. J. (2) 38, 417 (1986).

[38] L. Thomas and S. Wassell, J. Math. Phys. 36, 5480 (1995).

[39] C. Villegas-Blas, preprint.

[40] R. Szőke, Nagoya Math. J. 154, 171 (1999).

[41] S. De Bièvre, J. Math. Phys. 30, 1401 (1989).

[42] C. Isham and J. Klauder, J. Math. Phys. 32, 607 (1991).

[43] B. Torresani, Signal Processing 43, 341 (1995). 
Department of Mathematics, University of Notre Dame, Notre Dame, IN 46556, USA

E-mail address: bhall@nd.edu

Department of Mathematics, Baylor University, Waco, TX 76798, USA

E-mail address: jeffrey_mitchell@baylor.edu 